Published in final edited form as:

Synthesis (Stuttg). 2017 July ; 49(13): 2873-2888. doi:10.1055/s-0036-1589002.

\title{
Synthesis of 2-Alkenyl-Tethered Anilines
}

\author{
Scott E Denmark and Hyung Min Chi \\ Roger Adams Laboratory, Department of Chemistry, University of Illinois, Urbana, IL 61801, USA
}

\begin{abstract}
Three general routes for the synthesis of (E)-2-alkenyl-tethered anilines have been developed. The first route involves a 3-aza-Cope rearrangement of $N$-allylic anilines in the presence of a Lewis acid. The requisite $N$-allylic anilines were prepared by the addition of vinyl-magnesium reagents to the corresponding aldimines. The second route details a direct cross-metathesis of 2-allylic or 2homoallylic anilines with styrenes. The third route involves a palladium-catalyzed $\mathrm{C}-\mathrm{N}$ crosscoupling of aryl halides. Taken together, these three strategies allowed access to the requisite aniline substrates with pendant alkenes at the 2-position with excellent trans selectivities.
\end{abstract}

\section{Graphical abstract}

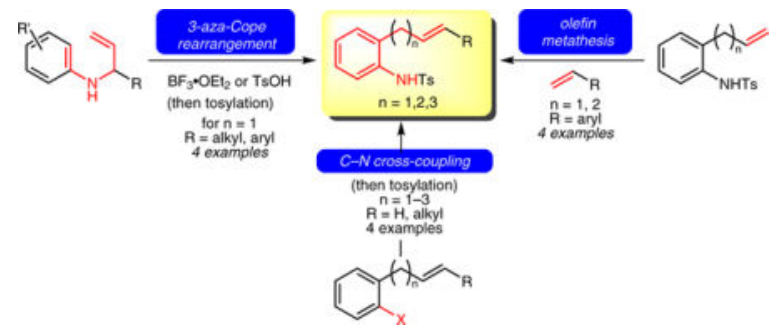

\section{Keywords}

2-alkenylanilines; 3-aza-Cope rearrangement; olefin metathesis; $\mathrm{C}$; $\mathrm{N}$ cross-coupling; trans olefins

Recent studies in these laboratories have described the catalytic, enantioselective, intramolecular sulfenoamination of 2-alkenyl-tethered anilines ${ }^{1}$ as a general method to access enantioenriched $\mathrm{N}$-containing heterocycles (Scheme 1), such as indolines, tetrahydroquinolines, and tetrahydrobenzazepines, which represent important classes of compounds exhibiting a range of biological activities. ${ }^{2}$

In the course of the reaction development, $N$-tosyl-protected anilines with pendant olefins at the 2-position were identified as viable substrates for the sulfenoamination process. Specifically, these reactions were found to be highly enantioselective and site-selective with $E$-disubstituted and terminal alkenes. ${ }^{1}$ Therefore, a rapid and versatile protocol for the preparation of these 2-alkenyl-substituted anilines was necessary. As no single method could

Correspondence to: Scott E Denmark.

Supporting Information

Supporting information for this article is available online at http://dx.doi.org/10.1055/s-0036-1589002. 
provide all of the different substitution patterns and tether lengths, developing a number of different methods to arrive at the substrates was necessary. Specifically, three different routes were developed that control the geometry of the double bond in the tether and the introduction of the amino group by rather different means, namely: (1) vinylmagnesium chloride addition to imines followed by 3 -aza-Cope rearrangement of the resulting $\mathrm{N}$-allylic anilines, (2) cross-metathesis of 2-substituted anilines bearing a terminal alkene, and (3) palladium-catalyzed C-N coupling of 2-substituted halobenzenes (Scheme 2).

Through application of these three strategies, twelve 2-alkenylaniline compounds 1a-l were prepared as substrates for the sulfenoamination reaction (Figure 1). 2-Allylic anilines 1a-d were synthesized by the addition of vinylmagnesium chloride followed by a tandem 3-azaCope rearrangement. While the preparations of $\mathbf{1 e}-\mathbf{g}$ were also viable through the first route, those compounds are also accessible by a more direct olefin cross-metathesis reaction that requires only a single step. 2-Homoallylic aniline $\mathbf{1 h}$ was also prepared via the second route, showing its ability to derivatize alkenylanilines with longer chains. Compounds $\mathbf{1} \mathbf{i}-\mathbf{l}$ were made by $\mathrm{C}-\mathrm{N}$ cross-coupling to demonstrate its complementary ability to access dialkylsubstituted alkene substrates with different tether lengths.

\section{3-Aza-Cope Rearrangement}

The aza-Cope rearrangement is a heteroatom variant of the Cope rearrangement, replacing a carbon with a nitrogen atom in one of the allyl components (Scheme 3). ${ }^{3}$ This reaction is often referred to as an amino-Claisen or an aza-Claisen rearrangement because of its structural similarity to the Claisen rearrangement.

The 3-aza-Cope rearrangement reactions have received less attention than Cope or Claisen rearrangements owing to the higher reaction temperatures needed, which inevitably leads to the formation of side products by thermal decomposition. ${ }^{3}$ Much effort has been invested to lower the activation barrier for the rearrangement by employing Brønsted or Lewis acids. ${ }^{4}$ Ward and co-workers were able to promote the rearrangement of 1,1-disubstituted $\mathrm{N}$-allylic anilines at significantly reduced temperature $\left(65^{\circ} \mathrm{C}\right)$ through the addition of a catalytic amount of toluenesulfonic acid (Scheme 4). ${ }^{4 \mathrm{a}}$

In our case, the 2-cinnamylanilines 5 were synthesized by 3 -aza-Cope rearrangement of asubstituted allylic anilines $\mathbf{3}$, which were prepared by the addition of vinylmagnesium chloride to the corresponding imines $\mathbf{2}$. This [3,3]-sigmatropic rearrangement was especially attractive due to its inherent high stereospecificity, furnishing trans-alkenes exclusively. This three-step sequence (imine formation/Grignard addition/rearrangement) was very effective for the synthesis of 2-cinnamylaniline derivatives.

The imines 2 were prepared by a straightforward condensation of the appropriate anilines and aldehydes, ${ }^{5}$ which was followed by a subsequent addition of vinylmagnesium chloride in the presence of zinc chloride to afford a-substituted allylic anilines $\mathbf{3}$ (Table 1). ${ }^{6}$ Initially, vinylmagnesium bromide was tested with zinc bromide as an additive, but no product formation was observed (entry 1). Interestingly, a slight excess (1.3 equiv) of the vinylmagnesium chloride was insufficient and resulted in production of only reduction 
product $4 \mathbf{a}$ (entry 2). ${ }^{6}$ However, successful addition of the vinyl moiety to the aldimines 2 was observed with 2 equivalents of the Grignard reagent (entries 3-5).

a-Substituted allylic anilines $\mathbf{3}$ were then subjected to aza-Cope rearrangement conditions (Table 2). When a-aryl-substituted allylic aniline $\mathbf{3 a}$ was treated with boron trifluoridediethyl ether complex, it rearranged to afford the desired trans-alkene 5a upon heating (Conditions A, entry 1). ${ }^{7}$ However, because of the unsatisfying yield of the rearrangement with generation of many side products, a simplified reaction protocol was employed. $p$ Toluenesulfonic acid catalyzed the 3-aza-Cope rearrangement of a-aryl-substituted allylic aniline 3a under much milder reaction conditions (Conditions B, entry 2). ${ }^{4 a}$ Other allylic anilines $\mathbf{3 b}$ and $\mathbf{3} \mathbf{c}$ also successfully rearranged to $\mathbf{5 b}$ and $\mathbf{5 c}$, respectively (entries 3 and 4 ). Protection of the resulting primary aniline with tosyl chloride afforded the desired substrates 1a, 1b, and 1c.

A similar strategy was employed for the construction of the isopropyl-substituted allylic aniline (Scheme 5). Aniline and isobutyraldehyde were first condensed to afford aldimine 2d, which was subsequently treated with vinylmagnesium chloride and 1(trimethylsilyl)benzotriazole using Katritzky's 1,2-addition protocol for imines bearing acidic a-protons. ${ }^{8}$ The resulting a-substituted allylic aniline $\mathbf{3 d}$ was then treated with boron trifluoride-diethyl ether complex to generate the expected primary aniline $\mathbf{5 d}$, which was subsequently protected with tosyl chloride to furnish tosylamide 1d. Interestingly, in this case $p$-toluenesulfonic acid failed to promote the rearrangement.

\section{Olefin Cross-Metathesis}

Olefin cross-metathesis is arguably one of the most powerful methods of olefin synthesis in modern organic chemistry. ${ }^{9}$ This process redistributes the double bonds of olefins allowing the formation of more highly functionalized olefins from simple alkene precursors. Pronounced functional group compatibility and mild reaction conditions render these reactions highly valuable and contribute to their wide application in many fields including polymer chemistry and natural product synthesis. ${ }^{10}$

Cross-metathesis of precursors bearing terminal alkenes was attempted to access disubstituted substrates, which would allow a one-step synthesis of diverse alkene substitution patterns and tether lengths. $N$-Tosyl-2-(2-propenyl)aniline (6) ${ }^{11}$ and $N$-tosyl-2(3-butenyl)aniline (7) ${ }^{12}$ were combined with several terminal olefins in the presence of a number of different ruthenium catalysts (Scheme 6). Although styrenyl products were formed with high $E$-selectivity, dialkyl-substituted products were formed as mixtures of geometrical isomers.

Next, the Grubbs-Hoveyda $2^{\text {nd }}$ generation catalyst was examined for an initial survey of cross-metathesis, but a rapid homodimerization of styrenes was observed with no desired product (Table 3, entry 1). In contrast, Grubbs $1^{\text {st }}$ generation indenylidene catalyst showed strong cross-metathesis by affording trans-olefin $1 \mathrm{e}$ with excellent geometrical selectivity (entry 2). Despite the excess amount of styrene added, substrate olefin 6 still remained after 18 hours. Extended reaction times showed a slight increase in yield (entry 3 ). Whereas 
metathesis with styrene afforded trans-olefin exclusively, metathesis with 1-pentene gave approximately a 2:1 mixture of trans- and cis-disubstituted olefins (entry 4). Neohexene, which has significant steric bulk adjacent to the olefin, was stable under the metathesis conditions (entry 5). Interestingly, the reaction with 4-pen-tenenitrile failed to give any products (entry 6). This reaction was repeated with the more reactive Grubbs-Hoveyda $2^{\text {nd }}$ generation catalyst to promote metathesis, and higher temperature was needed to initiate the reaction (entry 7). However, a complex mixture of $E$ - and $Z$-olefins was obtained along with some olefin isomerization products.

Encouraged by the positive screening result with styrene, the reaction was repeated on a gram scale which afforded the desired 2-cinnamyl- $N$-tosylaniline (1e) with the expected trans selectivity (Table 4, entry 1). In a similar manner, other styrenes were also examined. Metathesis with 4-vinylanisole and 4-bromostyrene produced the 2-cinnamylaniline derivatives $\mathbf{1 f}$ and $\mathbf{1 g}$, respectively, in excellent selectivities (entries 2 and 3). Since these aniline compounds $\mathbf{1 f}, \mathbf{1 g}$ can also be accessed through the aza-Cope rearrangement route, cross-metathesis of 2-homoal-lylaniline 7 was performed to demonstrate the advantage of this strategy (entry 4). Gratifyingly, the cross-metathesis of olefin 7 and styrene furnished longer-tethered compound $\mathbf{1 h}$ in good yield with exclusive trans selectivity.

\section{Palladium-Catalyzed Amination}

Although the aza-Cope rearrangement approach was successful for the preparation of aliphatic alkene tethers, the scope is limited to one-methylene-tethered substrates. For longer tethers, olefin metathesis is suitable for styrenes, but aliphatic alkenes were formed in poor geometrical selectivity. Accordingly, a different route was needed for the aliphatic substituents.

The synthesis of aniline derivatives has been tremendously facilitated by advances in transition-metal-catalyzed coupling of aryl halides with various nitrogen sources. The palladium-catalyzed $\mathrm{C}-\mathrm{N}$ cross-coupling strategy was additionally appealing because the requisite, 2-substituted phenols were readily available with high geometrical purity. ${ }^{13}$ The phenols could then be converted into anilines in two simple steps by either a direct installation of the tosylamide or by an amination followed by a tosyl protection (Scheme 7). 14

Between these two routes, the palladium-catalyzed $\mathrm{C}-\mathrm{N}$ coupling of aryl nonaflates with tosylamide appeared more attractive because the starting phenols are readily available (Scheme 7, a). ${ }^{14 a}$ However, whereas the nonaflate derived from $o$-cresol could be converted into the tosylaniline in excellent yield, this approach failed to afford any product for substrates bearing an ortho-tethered olefin (Scheme 8). This failure suggested a possible inhibition through binding of the palladium catalyst by the alkene.

In the alternative route, aryl halides are cross-coupled with ammonium sulfate under palladium catalysis to generate $N$-unsubstituted anilines, which can be subsequently tosylated (Scheme $7, \mathrm{~b}) .{ }^{14 \mathrm{~b}}$ This strategy was suitable for the synthesis of two- and threemethylene-tethered substrates. 
The configuration of the double bond in the 2-substituted haloarene was established with a Knoevenagel-Doebner condensation of malonic acid with 3-(2-chlorophenyl)propanal (8) to afford the nonconjugated carboxylic acid $\mathbf{9}$ (Scheme 9). ${ }^{15}$ Then, reduction of 9 to primary alcohol 10, ${ }^{15 a}$ followed by mesylation and displacement with cyanide, furnished nitrile intermediate $\mathbf{1 1}$ for the aryl amination. Aryl chloride $\mathbf{1 1}$ was coupled with ammonium sulfate to form aniline $\mathbf{1 2}$ following the palladium-catalyzed $\mathrm{C}-\mathrm{N}$ coupling developed by Hartwig and co-workers. ${ }^{14 \mathrm{~b}}$ The desired product was generated in modest yield along with isomerized side products. Tosylation of $\mathbf{1 2}$ afforded nitrile $\mathbf{1 i}$ which was reduced with $\mathrm{LiAlH}_{4}$ to the free amine. Subsequent tosyl protection of the amine furnished bistosylamide $\mathbf{1 j}$.

As was observed previously with the shorter tether, synthesis of the alkyl-substituted olefin side chain by cross-metathesis was only modestly trans-selective. A more selective synthesis of the trans-dialkyl-substituted alkene with a two-methylene tether entailed a Johnson orthoester Claisen rearrangement to set the configuration of the double bond in $\mathbf{1 k}$ (Scheme 10). Synthesis of the aniline substrate $1 \mathbf{k}$ was accomplished in a few steps from the alcohol intermediate 16, which was prepared by following a reported protocol by Wolfe and coworkers. ${ }^{16}$ Addition of vinyl-magnesium bromide to aldehyde $\mathbf{1 3}$ afforded allylic alcohol 14, which was followed by heating with triethyl orthoace-tate in the presence of a catalytic amount of acid to promote a [3,3]-sigmatropic rearrangement. ${ }^{16}$ Reduction of the resulting ester $\mathbf{1 5}$ to alcohol 16, ${ }^{16}$ followed by a two-step sequence of mesylation and hydride reduction, afforded the aryl bromide $17 .{ }^{17}$ The aryl bromide $\mathbf{1 7}$ was then subjected to Hartwig's C-N coupling method. ${ }^{14 \mathrm{~b}}$ Gratifyingly, in contrast to the one-methylene-tethered system, the reaction proceeded cleanly with no olefin migration observed. Tosyl protection of the resulting primary aniline $\mathbf{1 8}$ furnished the target substrate $\mathbf{1 k}$.

Encouraged by the satisfying results, the palladium-catalyzed $\mathrm{C}-\mathrm{N}$ coupling method was then applied to the preparation of even-longer-tethered substrates. 2-(4-Pent-enyl)aniline was prepared from 2-bromobenzyl bromide (19) by initial addition of 3-butenylmagnesium bromide with catalytic assistance of copper(I) iodide to afford the 2-pentenylaryl bromide $\mathbf{2 0}$ (Scheme 11). ${ }^{18}$ Hartwig's C-N coupling method was then employed to install the amine moiety, ${ }^{14 \mathrm{~b}}$ and the resulting primary aniline $\mathbf{2 1}$ was protected with a tosyl group to furnish the target sulfonamide $\mathbf{1 1}$.

In conclusion, three routes to accomplish the trans-selective preparation of 2-olefin-tethered anilines have been developed. The first method combines $\mathrm{ZnCl}_{2}$-assisted Grignard addition to aldimines with 3-aza-Cope rearrangement to access single-methylene-tethered 2-olefinic anilines with high efficiency. The second method is a more direct olefin cross-metathesis approach, which is especially effective for styrenyl substrates. The third method involves a $\mathrm{C}-\mathrm{N}$ cross-coupling reaction that is complementary to the other methods, and allows access to dialkyl olefinic anilines containing longer tethers. These $\mathrm{N}$-tosylanilines are suitable substrates for general electrophilic addition reactions, specifically designed for the enantioselective, catalytic sulfenoamination reaction that has been recently developed.

All reactions were performed in oven-dried $\left(140^{\circ} \mathrm{C}\right)$ and/or flame-dried glassware under an atmosphere of dry argon, unless otherwise noted. Column chromatography was performed 
using Merck silica gel 60 (40-63 $\mu \mathrm{m}$ particle size) purchased from Aldrich. Analytical TLC was performed on Merck silica gel plates with QF-254 indicator. $R_{f}$ values reported were measured using $10 \times 2 \mathrm{~cm}$ TLC plates in a developing chamber containing the solvent system described. Visualization was accomplished with UV light $(254 \mathrm{~nm})$ and/or $\mathrm{KMnO}_{4}$. Boiling points for Kugelrohr distillations correspond to corrected air bath temperatures. Melting points were determined in sealed tubes under vacuum on a Thomas Hoover capillary melting point apparatus, and are corrected. IR spectra were recorded on a PerkinElmer FT-IR system. ${ }^{1} \mathrm{H},{ }^{13} \mathrm{C}$, and ${ }^{19} \mathrm{~F}$ NMR spectra were recorded on an Inova spectrometer $\left(500 \mathrm{MHz},{ }^{1} \mathrm{H} ; 126 \mathrm{MHz},{ }^{13} \mathrm{C} ; 470 \mathrm{MHz},{ }^{19} \mathrm{~F}\right) .{ }^{1} \mathrm{H}$ and ${ }^{13} \mathrm{C}$ NMR spectra were acquired in $\mathrm{CDCl}_{3}$ referenced to residual $\mathrm{CHCl}_{3}$ at 7.26 and $77.00 \mathrm{ppm}$, respectively. Assignments were obtained by reference to COSY, HSQC, and HMBC correlations; atom numbering is shown in the Supporting Information. Mass spectrometry was performed by the University of Illinois Mass Spectrometry Center. ESI mass spectra were performed on a Waters or Micromass Q-Tof Ultima instrument. EI mass spectra were performed on a 70-VSE instrument. Elemental analyses were performed by the University of Illinois Microanalytical Service Laboratory and Robertson Microlit Laboratories, Inc.

Reaction solvents THF (Fisher, HPLC grade), $\mathrm{Et}_{2} \mathrm{O}$ (Fisher, BHT-stabilized ACS grade), and $\mathrm{CH}_{2} \mathrm{Cl}_{2}$ (Fisher, unstabilized HPLC grade) were dried by passage through two columns of neutral alumina in a solvent dispensing system. Reaction solvent toluene (Fischer, ACS grade) was dried by percolation through a column packed with neutral alumina and a column packed with Q5 reactant, a supported copper catalyst for scavenging oxygen, under a positive pressure of argon. Reaction solvent DMF (Fisher, HPLC grade) was dried by percolation through a column packed with molecular sieves in a solvent dispensing system. Solvents for chromatography, filtration, and recrystallization $\left[\mathrm{CH}_{2} \mathrm{Cl}_{2}\right.$ (Aldrich, ACS grade), EtOAc (Fisher, ACS grade), $\mathrm{Et}_{2} \mathrm{O}$ (Fisher, ACS grade), hexanes (Fisher, Optima)] were used as received. $\mathrm{Et}_{3} \mathrm{~N}$ (Alfa Aesar) and pyridine (Fisher) were freshly distilled from $\mathrm{CaH}_{2}$. 'Brine' refers to a saturated aqueous solution of sodium chloride.

\section{Addition of Grignard Reagent to Aldimines (Table 1)}

\section{4-Fluoro- $\mathrm{N}$-(1-phenylallyl)aniline (3a) ${ }^{6}$}

To a flame-dried, 50-mL Schlenk flask was added a solution of vinyl-magnesium chloride in THF (18.8 mL, $1.6 \mathrm{M}, 30 \mathrm{mmol}, 2$ equiv) and a solution of $\mathrm{ZnCl}_{2}$ in THF ( $3 \mathrm{~mL}, 1.0 \mathrm{M}, 3$ mmol, 0.2 equiv). After the solution was stirred for $20 \mathrm{~min}$ at r.t., imine $\mathbf{2 a}^{5 \mathrm{a}}(2.99 \mathrm{~g}, 15$ mmol) was added under positive argon pressure. The solution was stirred at r.t. for $16 \mathrm{~h}$. The reaction was quenched with sat. aqueous $\mathrm{NH}_{4} \mathrm{Cl}$ solution $(30 \mathrm{~mL})$ and extracted with EtOAc $(3 \times 40 \mathrm{~mL})$. The organic layers were combined, washed with brine $(30 \mathrm{~mL})$, dried over $\mathrm{MgSO}_{4}$, and filtered. The filtrate was concentrated under reduced pressure $\left(30^{\circ} \mathrm{C}, 10\right.$ $\mathrm{mmHg}$ ) to afford $3.05 \mathrm{~g}$ of a dark brown oil. Purification by silica gel flash chromatography $(16 \times 5 \mathrm{~cm} ; 800 \mathrm{~mL}$ of 93:5:2 hexane/EtOAc/Et $3 \mathrm{~N}, 200 \mathrm{~mL}$ of 90:10 hexane/EtOAc, 200 $\mathrm{mL}$ of 85:15 hexane/EtOAc, $200 \mathrm{~mL}$ of 80:20 hexane/EtOAc) afforded $2.84 \mathrm{~g} \mathrm{(83 \% )}$ of 3a as a brown liquid; $R_{f}=0.54$ (hexanes/EtOAc, 9:1) [UV]. The spectroscopic data matched those reported previously. ${ }^{19}$ 
IR (neat): 3413 (w), 3029 (w), 1612 (w), 1506 (s), 1452 (m), 1401 (w), 1312 (m), 1217 (s), 1156 (m), 1139 (w), 1108 (w), 1066 (w), 1028 (w), 991 (w), 817 (s), 779 (m), 748 (m) cm cm. $^{-1}$.

${ }^{1} \mathrm{H}$ NMR (500 MHz, CDCl $): \delta=7.46-7.38(\mathrm{~m}, 4 \mathrm{H}, \mathrm{HC}(10,11)), 7.34(\mathrm{tt}, J=6.5,1.5 \mathrm{~Hz}, 1$ H, HC(12)), 6.93-6.87 (m, 2 H, HC(7)), 6.61-6.55 (m, 2 H, HC(6)), 6.09 (ddd, $J=16.5$, 10.0, $6.0 \mathrm{~Hz}, 1 \mathrm{H}, \mathrm{HC}(4))$, 5.37-5.26 (m, $2 \mathrm{H}, \mathrm{HC}(5)), 4.93$ (d, J=6.0 Hz, $1 \mathrm{H}, \mathrm{HC}(3)), 3.99$ (s, $1 \mathrm{H}, \mathrm{HN}(2)$ ).

${ }^{13} \mathrm{C}$ NMR (126 MHz, $\left.\mathrm{CDCl}_{3}\right): \delta=155.8(\mathrm{~d}, J=235.2 \mathrm{~Hz}, \mathrm{C} 8), 143.5(\mathrm{C} 1), 141.7$ (C9), 139.0 (C4), 128.7 (C10), 127.5 (C12), 127.0 (C11), 116.1 (C5), 115.5 (C7), 114.4 (C6), 61.5 (C3).

${ }^{19} \mathrm{~F}$ NMR (470 MHz, $\left.\mathrm{CDCl}_{3}\right): \delta=-128.2$.

MS (ESI): $m / z(\%)=117(27), 145(24), 183(16), 200(18), 228(12)[\mathrm{M}+\mathrm{H}]^{+}, 280(15)$, 290 (100), 291 (32), 317 (24), 342 (15), 370 (21), 404 (19).

HRMS (ESI): $m / z[\mathrm{M}+\mathrm{H}]^{+}$calcd for $\mathrm{C}_{15} \mathrm{H}_{15} \mathrm{FN}$ : 228.1189; found: 228.1197 .

\section{4-Methoxy- $N-(1-p h e n y l a l l y l) a n i l i n e ~(3 b)^{6}$}

To a flame-dried, 100-mL Schlenk flask was added a solution of vinylmagnesium chloride in THF (18.8 mL, 1.6 M, $30 \mathrm{mmol}, 2$ equiv) and a solution of $\mathrm{ZnCl}_{2}$ in THF ( $3 \mathrm{~mL}, 1.0 \mathrm{M}, 3$ mmol, 0.2 equiv). After the solution was stirred for $20 \mathrm{~min}$ at r.t., imine $2 \mathbf{b}^{5 \mathrm{~b}}$ ( $3.17 \mathrm{~g}, 15$ mmol) was added under positive argon pressure. The solution was stirred at r.t. for $16 \mathrm{~h}$. The reaction was quenched with sat. aqueous $\mathrm{NH}_{4} \mathrm{Cl}$ solution $(30 \mathrm{~mL})$ and extracted with EtOAc $(3 \times 30 \mathrm{~mL})$. The organic layers were combined, washed with brine $(30 \mathrm{~mL})$, dried over $\mathrm{MgSO}_{4}$, and filtered. The filtrate was concentrated under reduced pressure $\left(30{ }^{\circ} \mathrm{C}, 10\right.$ $\mathrm{mmHg})$ to afford a dark brown oil. Purification by silica gel flash chromatography $(18 \times 5$ $\mathrm{cm} ; 1000 \mathrm{~mL}$ of 93:5:2 hexane/EtOAc/Et $3 \mathrm{~N}, 500 \mathrm{~mL}$ of $95: 5$ hexane/EtOAc, $300 \mathrm{~mL}$ of 93:7 hexane/EtOAc, $300 \mathrm{~mL}$ of 90:10 hexane/EtOAc) afforded $2.93 \mathrm{~g}(82 \%)$ of $\mathbf{3 b}$ as a brown liquid; $R_{f}=0.44$ (hexanes/EtOAc, 4:1) [UV]. The spectroscopic data matched those reported previously. 19

IR (neat): 3394 (w), 3027 (w), 2831 (w), 1617 (w), 1508 (s), 1463 (m), 1451 (m), 1442 (w), $1406(\mathrm{w}), 1308(\mathrm{w}), 1290(\mathrm{w}), 1240(\mathrm{~s}), 1229(\mathrm{~s}), 1178(\mathrm{~m}), 1156(\mathrm{w}), 1139(\mathrm{w}), 1114(\mathrm{w})$, 1094 (w), 1034 (m), 991 (w), 923 (m), 817 (s), 764 (m), $746(\mathrm{~m}) \mathrm{cm}^{-1}$.

${ }^{1} \mathrm{H}$ NMR $\left(500 \mathrm{MHz}, \mathrm{CDCl}_{3}\right): \delta=7.42-7.32(\mathrm{~m}, 4 \mathrm{H}, \mathrm{HC}(10,11), 7.31-7.24(\mathrm{~m}, 1 \mathrm{H}$, HC(12)), 6.78-6.72 (m, 2 H, HC(7)), 6.61-6.54 (m, 2 H, HC(6)), 6.04 (ddd, J= 17.2, 10.2, $6.0 \mathrm{~Hz}, 1 \mathrm{H}, \mathrm{HC}(4)), 5.32-5.19$ (m, $2 \mathrm{H}, \mathrm{HC}(5)), 4.87$ (d, $J=6.1 \mathrm{~Hz}, 1 \mathrm{H}, \mathrm{HC}(3)), 3.82$ (s, 1 $\mathrm{H}, \mathrm{HN}(2)), 3.73$ (s, $3 \mathrm{H}, \mathrm{HC}(13)$ ).

${ }^{13} \mathrm{C}$ NMR (126 MHz, $\mathrm{CDCl}_{3}$ ): $\delta=152.4(\mathrm{C} 8), 142.3(\mathrm{C} 9), 141.7$ (C1), 139.7 (C4), 128.9 (C11), 127.6 (C12), 127.4 (C10), 116.1 (C6), 115.1 (C5), 114.9 (C7), 61.0 (C3), 55.9 (C13).

MS (ESI): $\mathrm{m} / z(\%)=117(33), 217(25), 240(75)[\mathrm{M}+\mathrm{H}]^{+}, 241$ (13), 262 (22), $270(21)$, 328 (100), 329 (29), 371 (96), 372 (31), 479 (23). 
HRMS (ESI): $m / z[\mathrm{M}+\mathrm{H}]^{+}$calcd for $\mathrm{C}_{16} \mathrm{H}_{18} \mathrm{NO}$ : 240.1388; found: 240.1393 .

\section{$N$-(1-Phenylallyl)naphthalen-2-amine (3c)}

To a flame-dried, 50-mL Schlenk flask was added a solution of vinyl-magnesium chloride in THF (18.8 mL, $1.6 \mathrm{M}, 30 \mathrm{mmol}, 2$ equiv) and a solution of $\mathrm{ZnCl}_{2}$ in THF ( $3 \mathrm{~mL}, 1.0 \mathrm{M}, 3$ mmol, 0.2 equiv). After the solution was stirred for $20 \mathrm{~min}$ at r.t., imine $2 \mathrm{c}^{5 \mathrm{c}}(3.47 \mathrm{~g}, 15$ mmol) was added under positive argon pressure. The solution was stirred at r.t. for $16 \mathrm{~h}$. The reaction was quenched with sat. aqueous $\mathrm{NH}_{4} \mathrm{Cl}$ solution $(30 \mathrm{~mL})$ and extracted with EtOAc $(3 \times 40 \mathrm{~mL})$. The organic layers were combined, washed with brine $(30 \mathrm{~mL})$, dried over $\mathrm{MgSO}_{4}$, and filtered. The filtrate was concentrated under reduced pressure $\left(30^{\circ} \mathrm{C}, 10\right.$ $\mathrm{mmHg}$ ) to afford $3.05 \mathrm{~g}$ of a dark brown oil. Purification by silica gel flash chromatography $\left(16 \times 5 \mathrm{~cm} ; 800 \mathrm{~mL}\right.$ of 93:5:2 hexane/EtOAc/ $\mathrm{Et}_{3} \mathrm{~N}, 200 \mathrm{~mL}$ of 90:10 hexane/EtOAc, 200 $\mathrm{mL}$ of 85:15 hexane/EtOAc, $200 \mathrm{~mL}$ of 80:20 hexane/EtOAc) afforded $3.70 \mathrm{~g} \mathrm{(95 \% )} \mathrm{of} \mathrm{3c}$ as a brown liquid; $R_{f}=0.35$ (hexanes/EtOAc, 4:1) [UV]. The spectroscopic data matched those reported previously. ${ }^{20}$

IR (neat): 3409 (w), 3056 (w), 3026 (w), 2847 (w), 1627 (s), 1601 (m), 1519 (s), 1478 (m), 1451 (m), 1424 (w), 1396 (m), 1358 (m), 1301 (w), 1265 (w), 1223 (m), 1188 (m), 1157 (w), 1145 (w), 1125 (w), 1094 (w), 1066 (w), 1028 (w), 1018 (w), 990 (w), 967 (w), 954 (w), $924(\mathrm{~m}), 909(\mathrm{~m}), 865(\mathrm{w}), 827$ (s), $806(\mathrm{~s}), 743(\mathrm{~s}) \mathrm{cm}^{-1}$.

${ }^{1} \mathrm{H}$ NMR $\left(500 \mathrm{MHz}, \mathrm{CDCl}_{3}\right): \delta=7.65(\mathrm{~d}, J=8.1 \mathrm{~Hz}, 1 \mathrm{H}, \mathrm{HC}(11)), 7.62(\mathrm{~d}, J=8.8 \mathrm{~Hz}, 1$ H, HC(13)), 7.54 (dd, $J=8.3,1.1 \mathrm{~Hz}, 1 \mathrm{H}, \mathrm{HC}(8)), 7.54$ (d, $J=8.2 \mathrm{~Hz}, 1 \mathrm{H}, \mathrm{HC}(16)), 7.43$ (d, $J=7.5 \mathrm{~Hz}, 2 \mathrm{H}, \mathrm{HC}(16)), 7.45-7.41$ (m, $2 \mathrm{H}, \mathrm{HC}(17)), 7.37$ (t, $J=7.6 \mathrm{~Hz}, 1 \mathrm{H}, \mathrm{HC}(9))$, 7.34-7.27 (m, $1 \mathrm{H}, \mathrm{HC}(18)$ ), 7.18 (ddd, $J=8.1,6.8,1.2 \mathrm{~Hz}, 1 \mathrm{H}, \mathrm{HC}(10)), 6.92$ (dd, $J=8.8$, $2.4 \mathrm{~Hz}, 1 \mathrm{H}, \mathrm{HC}(14)), 6.77$ (d, $J=2.4 \mathrm{~Hz}, 1 \mathrm{H}, \mathrm{HC}(6)), 6.10$ (ddd, $J=17.1,10.2,5.8 \mathrm{~Hz}, 1$ H, HC(4)), 5.33 (dt, $J=17.1,1.4 \mathrm{~Hz}, 1 \mathrm{H}, \mathrm{HC}(5)), 5.27$ (dt, $J=10.2,1.3 \mathrm{~Hz}, 1 \mathrm{H}, \mathrm{HC}(5)$ ), $5.08(\mathrm{t}, J=5.5 \mathrm{~Hz}, 1 \mathrm{H}, \mathrm{HC}(3)), 4.22(\mathrm{~d}, J=5.2 \mathrm{~Hz}, 1 \mathrm{H}, \mathrm{HN}(2))$.

${ }^{13} \mathrm{C}$ NMR (126 MHz, $\left.\mathrm{CDCl}_{3}\right): \delta=144.7(\mathrm{C} 1), 141.6(\mathrm{C} 15), 138.7$ (C4), 134.9 (C7), 128.8 (C13,17), 127.6 (C11), 127.5 (C18), 127.5 (C12), 127.2 (C16), 126.2 (C9), 126.0 (C8), 122.1 (C10), 118.1 (C14), 116.2 (C5), 105.9 (C6), 60.8 (C3).

MS (ESI): $m / z(\%)=117(100), 156(63), 234$ (48), $260(60)[\mathrm{M}+\mathrm{H}]^{+}, 274(31), 400(20)$, 415 (19), 517 (34).

HRMS (ESI): $m / z$ [M $+\mathrm{H}]^{+}$calcd for $\mathrm{C}_{19} \mathrm{H}_{18} \mathrm{~N}$ : 260.1439; found: 260.1445 .

\section{Preparation of 2-Alkenylanilines by 3-Aza-Cope Rearrangement (Table 2) $(E)-2-C i n n a m y l-4-f l u o r o a n i l i n e ~(5 a)^{4 a}$}

To a 500-mL Schlenk flask equipped with a magnetic stir bar were added 4-fluoro- $\mathrm{N}$-(1phenylallyl)aniline (3a) (2.05 g, $9.0 \mathrm{mmol})$, TsOH$\cdot \mathrm{H}_{2} \mathrm{O}$ (352 mg, $1.8 \mathrm{mmol}, 0.2$ equiv), and a mixed solution of $\mathrm{MeCN}(90 \mathrm{~mL})$ and $\mathrm{H}_{2} \mathrm{O}(10 \mathrm{~mL})$. The solution was heated to $65^{\circ} \mathrm{C}$ in an oil bath while the reaction was monitored by NMR spectroscopy (the reaction stalled after $36 \mathrm{~h}$ ). Volatiles were removed under reduced pressure and the residue was extracted 
with $\mathrm{Et}_{2} \mathrm{O}(3 \times 30 \mathrm{~mL})$. The combined organic layer was washed with $1 \mathrm{M} \mathrm{NaOH}(30 \mathrm{~mL})$ and $\mathrm{H}_{2} \mathrm{O}(30 \mathrm{~mL})$, dried over $\mathrm{Na}_{2} \mathrm{SO}_{4}$, and concentrated under reduced pressure $\left(30{ }^{\circ} \mathrm{C}, 10\right.$ $\mathrm{mmHg})$. Purification of the residue by silica gel flash chromatography $(9.5 \times 5 \mathrm{~cm} ; 500 \mathrm{~mL}$ of 88:10:2 hexane/EtOAc/ $\mathrm{Et}_{3} \mathrm{~N}, 200 \mathrm{~mL}$ of 90:10 hexane/EtOAc, $200 \mathrm{~mL}$ of 80:20 hexane/ EtOAc, $250 \mathrm{~mL}$ of 50:50 hexane/EtOAc) afforded $1.45 \mathrm{~g}(71 \%)$ of $\mathbf{5 a}$ as a brown liquid; $R_{f}$ $=0.26($ hexanes/EtOAc, 4:1) [UV]. The spectroscopic data matched those reported previously. ${ }^{20}$

IR (neat): 3444 (w), 3367 (w), 3032 (w), 1624 (m), 1610 (w), 1596 (w), 1577 (w), 1494 (s), 1448 (w), 1436 (s), 1420 (m), 1352 (w), 1332 (w), 1277 (w), 1261 (m), 1197 (m), 1144 (m), $1081(\mathrm{w}), 1070(\mathrm{w}), 1055(\mathrm{w}), 1028(\mathrm{w}), 990(\mathrm{~m}), 983(\mathrm{~m}), 972(\mathrm{w}), 951(\mathrm{~s}), 859(\mathrm{~s}), 822$ (m), $807(\mathrm{~s}), 751(\mathrm{~s}) \mathrm{cm}^{-1}$.

${ }^{1} \mathrm{H} \mathrm{NMR}\left(500 \mathrm{MHz}, \mathrm{CDCl}_{3}\right): \delta=7.45-7.23(\mathrm{~m}, 5 \mathrm{H}, \mathrm{HC}(11,12,13)), 6.88$ (ddt, $J=19.9$, $8.5,2.7 \mathrm{~Hz}, 2 \mathrm{H}, \mathrm{HC}(2,6)), 6.65$ (dd, $J=8.7,4.9 \mathrm{~Hz}, 1 \mathrm{H}, \mathrm{HC}(3)), 6.50$ (d, $J=15.9 \mathrm{~Hz}, 1 \mathrm{H}$, $\mathrm{HC}(9)), 6.35$ (dtd, $J=15.8,6.3,1.8 \mathrm{~Hz}, 1 \mathrm{H}, \mathrm{HC}(8)), 3.59$ (s, $2 \mathrm{H}, \mathrm{HN}(14)), 3.45$ (d, $J=6.3$ $\mathrm{Hz}, 2 \mathrm{H}, \mathrm{HC}(7))$.

${ }^{13} \mathrm{C}$ NMR (126 MHz, $\left.\mathrm{CDCl}_{3}\right): \delta=156.7(\mathrm{~d}, J=235.9 \mathrm{~Hz}, \mathrm{C} 1), 141.1(\mathrm{C} 4), 137.3(\mathrm{C} 10)$, 132.0 (C13), 128.9 (C12), 127.8 (C9 or C11), 127.0 (C9 or C11), 126.5 (C8), 126.2 (d, $J=$ $6.6 \mathrm{~Hz}, \mathrm{C} 5), 116.9$ (d, $J=5.7 \mathrm{~Hz}, \mathrm{C} 3), 116.7$ (d, $J=20.4 \mathrm{~Hz}, \mathrm{C} 6), 114.1$ (d, $J=22.1 \mathrm{~Hz}$, $\mathrm{C} 2), 35.6(\mathrm{C} 7)$.

MS (ESI): $m / z(\%)=124(41), 228(100)[\mathrm{M}+\mathrm{H}]^{+}, 229(29)$.

HRMS (ESI): $m / z[\mathrm{M}+\mathrm{H}]^{+}$calcd for $\mathrm{C}_{15} \mathrm{H}_{15} \mathrm{FN}$ : 228.1189; found: 228.1192 .

\section{(E)-N-(2-Cinnamyl-4-fluorophenyl)-4-methylbenzenesulfonamide (1a) ${ }^{11}$}

To a flame-dried, 50-mL Schlenk flask equipped with a magnetic stir bar, purged with a positive pressure of argon, were added aniline $5 \mathbf{a}(1.13 \mathrm{~g}, 5.0 \mathrm{mmol})$ and $\mathrm{CH}_{2} \mathrm{Cl}_{2}(15 \mathrm{~mL}$, $0.3 \mathrm{M})$. The solution was cooled to $0{ }^{\circ} \mathrm{C}$ and pyridine $(2.0 \mathrm{~mL}, 25.0 \mathrm{mmol}, 5.0$ equiv) and $\mathrm{TsCl}(1.43 \mathrm{~g}, 7.5 \mathrm{mmol}, 1.5$ equiv) were added. The reaction mixture was warmed to r.t. and stirred for $12 \mathrm{~h}$. Then, brine $(30 \mathrm{~mL})$ was added and the solution was extracted with $\mathrm{CH}_{2} \mathrm{Cl}_{2}$ $(3 \times 30 \mathrm{~mL})$. The organic layers were combined, dried over $\mathrm{MgSO}_{4}$, filtered, and concentrated under reduced pressure $\left(30^{\circ} \mathrm{C}, 10 \mathrm{mmHg}\right)$. The crude solid was purified by silica gel flash chromatography $(8 \times 5 \mathrm{~cm} ; 500 \mathrm{~mL}$ of $90: 10$ hexane/EtOAc, $200 \mathrm{~mL}$ of $85: 15$ hexane/EtOAc, $200 \mathrm{~mL}$ of 80:20 hexane/EtOAc, $200 \mathrm{~mL}$ of 75:25 hexane/EtOAc, 200 $\mathrm{mL}$ of 50:50 hexane/EtOAc) to afford $1.88 \mathrm{~g}(98 \%)$ of a brown liquid. Crystallization of the brown liquid was achieved by dissolving in boiling EtOAc $(10 \mathrm{~mL})$ and cooling the solution to r.t. and then to $-20^{\circ} \mathrm{C}$ in a freezer. The $1^{\text {st }}$ crop was collected by filtration, and the mother liquor was concentrated and recrystallized (boiling EtOAc, $5 \mathrm{~mL}$ ). After cooling at $-20{ }^{\circ} \mathrm{C}$ and filtration, a combined yield of $1.63 \mathrm{~g}$ (86\%) of 1a was obtained; fluffy solid; mp 128$129^{\circ} \mathrm{C}$ (sealed tube); $R_{f}=0.32$ (hexanes/EtOAc, $4: 1$ ) [UV].

IR (neat): 3270 (w), 3028 (w), 2924 (w), 1614 (w), 1598 (w), 1494 (m), 1448 (w), 1435 (w), 1392 (w), 1328 (w), 1305 (w), $1273(\mathrm{w}), 1200$ (w), $1184(\mathrm{w}), 1157$ (s), $1120(\mathrm{w}), 1092(\mathrm{w})$,

Synthesis (Stuttg). Author manuscript; available in PMC 2018 June 20. 
$1019(\mathrm{w}), 963(\mathrm{w}), 904(\mathrm{w}), 814(\mathrm{w}), 754(\mathrm{w}), 730(\mathrm{w}), 706(\mathrm{w}), 692(\mathrm{w}), 664(\mathrm{~m}), 595(\mathrm{w})$, $548(\mathrm{~m}), 527(\mathrm{~m}), 493(\mathrm{w}) \mathrm{cm}^{-1}$.

${ }^{1} \mathrm{H}$ NMR (500 MHz, $\left.\mathrm{CDCl}_{3}\right): \delta=7.57(\mathrm{~d}, J=8.3 \mathrm{~Hz}, 2 \mathrm{H}, \mathrm{HC}(16)), 7.31-7.26(\mathrm{~m}, 6 \mathrm{H}$, $\mathrm{HC}(3,11,12,13)), 7.21$ (d, $J=8.1 \mathrm{~Hz}, 2 \mathrm{H}, \mathrm{HC}(17)), 6.89$ (ddd, $J=19.0,8.6,3.0 \mathrm{~Hz}, 2 \mathrm{H}$, $\mathrm{HC}(2,6)), 6.32$ (s, $1 \mathrm{H}, \mathrm{HN}(14)$ ), 6.27 (dt, $J=16.2,0.9 \mathrm{~Hz}, 1 \mathrm{H}, \mathrm{HC}(9)), 6.04$ (dt, $J=15.9$, $6.5 \mathrm{~Hz}, 1 \mathrm{H}, \mathrm{HC}(8)), 3.18$ (d, J=6.01 Hz, $2 \mathrm{H}, \mathrm{HC}(7)), 2.39$ (s, $3 \mathrm{H}, \mathrm{HC}(19)$.

${ }^{13} \mathrm{C}$ NMR (126 MHz, $\left.\mathrm{CDCl}_{3}\right): \delta=162.3(\mathrm{C} 1), 144.2(\mathrm{C} 18), 136.7$ (C15), $136.6(\mathrm{C} 4), 132.7$ (C9), 130.6 (C5 or C10), 130.5 (C5 or C10), 129.9 (C17), 128.8 (C6), 128.0 (d, J=6.0 Hz, C3), 127.9 (C12), 127.8 (C13), 127.4 (C16), 126.5 (C11), 126.4 (C8), 117.3 (d, J=22.7 Hz, C6), 114.6 (d, $J=22.2 \mathrm{~Hz}, \mathrm{C} 2), 35.2$ (C7), 21.8 (C19).

${ }^{19} \mathrm{~F}$ NMR $\left(470 \mathrm{MHz}, \mathrm{CDCl}_{3}\right): \delta=-115.2$.

MS (ESI): $m / z(\%)=226(21), 227(100)[\mathrm{M}-\mathrm{Ts}+\mathrm{H}]^{+}, 228(18), 382(72)[\mathrm{M}+\mathrm{H}]^{+}, 399$ (36), 404 (17).

HRMS (ESI): $m / z[\mathrm{M}+\mathrm{H}]^{+}$calcd for $\mathrm{C}_{22} \mathrm{H}_{21} \mathrm{FNO}_{2} \mathrm{~S}: 382.1271$; found: 382.1273 .

Anal. Calcd for $\mathrm{C}_{22} \mathrm{H}_{20} \mathrm{FNO}_{2} \mathrm{~S}$ (381.46): C, 69.27; H, 5.28; N, 3.67. Found: C, 69.36; H, $5.20 ; \mathrm{N}, 3.70$.

\section{(E)-2-Cinnamyl-4-methoxyaniline (5b) ${ }^{4 a}$}

To a 100-mL Schlenk flask equipped with a magnetic stir bar were added 4-methoxy- $N$-(1phenylallyl)aniline (3b) (239 mg, $1 \mathrm{mmol})$, Ts $\mathrm{OH} \cdot \mathrm{H}_{2} \mathrm{O}$ ( $39 \mathrm{mg}, 0.2 \mathrm{mmol}, 0.2$ equiv), and a mixed solution of $\mathrm{MeCN}(10 \mathrm{~mL})$ and $\mathrm{H}_{2} \mathrm{O}(1 \mathrm{~mL})$. The solution was heated to $65{ }^{\circ} \mathrm{C}$ in an oil bath while the reaction was monitored by NMR spectroscopy (the reaction stalled after $36 \mathrm{~h})$. Volatiles were removed under reduced pressure and the residue was extracted with $\mathrm{Et}_{2} \mathrm{O}(3 \times 10 \mathrm{~mL})$. The combined organic layer was washed with $1 \mathrm{M} \mathrm{NaOH}(10 \mathrm{~mL})$ and $\mathrm{H}_{2} \mathrm{O}(10 \mathrm{~mL})$, dried over $\mathrm{MgSO}_{4}$, and concentrated under reduced pressure $\left(30^{\circ} \mathrm{C}, 10\right.$ $\mathrm{mmHg})$. Purification of the residue by silica gel flash chromatography $(16 \times 2 \mathrm{~cm} ; 500 \mathrm{~mL}$ of 95:5 hexane/EtOAc with $10 \mathrm{~mL}$ of $\mathrm{Et}_{3} \mathrm{~N}, 200 \mathrm{~mL}$ of 90:10 hexane/EtOAc, $200 \mathrm{~mL}$ of 50:50 hexane/EtOAc) afforded $195 \mathrm{mg}(81 \%)$ of a brown liquid. Crystallization of the brown liquid was achieved by dissolving in boiling $\mathrm{Et}_{2} \mathrm{O}(0.5 \mathrm{~mL})$, followed by slow addition of pentane $(3 \mathrm{~mL})$. The solution was cooled to r.t. and then to $-20^{\circ} \mathrm{C}$ in a freezer. Filtration over glass wool afforded $170 \mathrm{mg}(71 \%)$ of $\mathbf{5 b}$; white needle-like crystals; mp 63$64{ }^{\circ} \mathrm{C}$ (sealed tube); $R_{f}=0.25$ (hexanes/EtOAc, 4:1) [UV].

IR (neat): 3358 (w), 3024 (w), 2998 (w), 2936 (w), 2906 (w), 2831 (w), 1607 (w), 1500 (s), $1465(\mathrm{~m}), 1448(\mathrm{~m}), 1432(\mathrm{~m}), 1323(\mathrm{w}), 1286(\mathrm{w}), 1242(\mathrm{~s}), 1211(\mathrm{w}), 1189(\mathrm{w}), 1154(\mathrm{~m})$, $1075(\mathrm{w}), 1040(\mathrm{~m}), 969(\mathrm{~m}), 940(\mathrm{w}), 870(\mathrm{w}), 854(\mathrm{w}), 811(\mathrm{~m}), 747(\mathrm{~m}), 732(\mathrm{~m}), 692(\mathrm{~s})$, $566(\mathrm{~m}), 498(\mathrm{w}), 475(\mathrm{~m}) \mathrm{cm}^{-1}$.

${ }^{1} \mathrm{H} \mathrm{NMR}\left(500 \mathrm{MHz}, \mathrm{CDCl}_{3}\right): \delta=7.35(\mathrm{~d}, J=7.0 \mathrm{~Hz}, 2 \mathrm{H}, \mathrm{HC}(11)), 7.31-7.27(\mathrm{~m}, 2 \mathrm{H}$, $\mathrm{HC}(12)), 7.24-7.17$ (m, $1 \mathrm{H}, \mathrm{HC}(13)), 6.72$ (d, $J=2.8 \mathrm{~Hz}, 1 \mathrm{H}, \mathrm{HC}(6)), 6.67$ (dd, $J=8.4$, $2.9 \mathrm{~Hz}, 1 \mathrm{H}, \mathrm{HC}(2)), 6.65$ (d, $J=8.5 \mathrm{~Hz}, 1 \mathrm{H}, \mathrm{HC}(3)), 6.45$ (dt, $J=16.0,1.4 \mathrm{~Hz}, 1 \mathrm{H}$, 
$\mathrm{HC}(9)), 6.33$ (dt, $J=15.8,6.2 \mathrm{~Hz}, 1 \mathrm{H}, \mathrm{HC}(8)), 3.75$ (s, $3 \mathrm{H}, \mathrm{HC}(14)), 3.45$ (d, $J=7.3 \mathrm{~Hz}, 4$ $\mathrm{H}, \mathrm{HC}(7)$ and $\mathrm{HN}(15))$.

${ }^{13} \mathrm{C}$ NMR (126 MHz, $\left.\mathrm{CDCl}_{3}\right): \delta=153.2(\mathrm{C} 1), 138.5$ (C4), $137.4(\mathrm{C} 5), 131.6$ (C9), 128.8 (C12), 127.7 (C8), 127.5 (C13), 126.4 (C11), 126.2 (C10), 117.3 (C3), 116.3 (C6), 113.0 (C2), 56.0 (C14), 36.0 (C7).

MS (ESI): $m / z(\%)=136(51)[\mathrm{M}-\text { styrene }+\mathrm{H}]^{+}, 239(12), 240(100)[\mathrm{M}+\mathrm{H}]^{+}, 241(17)$.

HRMS (ESI): $m / z$ [M + H] $]^{+}$calcd for $\mathrm{C}_{16} \mathrm{H}_{18} \mathrm{NO}$ : 240.1388; found: 240.1391 .

Anal. Calcd for $\mathrm{C}_{16} \mathrm{H}_{17} \mathrm{NO}$ (239.31): C, 80.30; H, 7.16; N, 5.85. Found: C, 80.25; H, 7.05; N, 5.93 .

\section{(E)-N-(2-Cinnamyl-4-methoxyphenyl)-4-methylbenzenesulfon-amide (1b) ${ }^{\mathbf{1 1}}$}

To a flame-dried, $25-\mathrm{mL}$ Schlenk flask equipped with a magnetic stir bar, purged with a positive pressure of argon, were added aniline $\mathbf{5 b}(1.17 \mathrm{~g}, 4.9 \mathrm{mmol})$ and $\mathrm{CH}_{2} \mathrm{Cl}_{2}(15 \mathrm{~mL}$, $0.3 \mathrm{M})$. The solution was cooled to $0{ }^{\circ} \mathrm{C}$ and pyridine $(1.97 \mathrm{~mL}, 24.5 \mathrm{mmol}, 5.0$ equiv) and $\mathrm{TsCl}(1.4 \mathrm{~g}, 7.35 \mathrm{mmol}, 1.5$ equiv) were added. The reaction mixture was warmed to r.t. and stirred for $12 \mathrm{~h}$. Then, brine $\left(30 \mathrm{~mL}\right.$ ) was added and the solution was extracted with $\mathrm{CH}_{2} \mathrm{Cl}_{2}$ $(3 \times 30 \mathrm{~mL})$. The organic layers were combined, dried over $\mathrm{MgSO}_{4}$, filtered, and concentrated under reduced pressure $\left(30^{\circ} \mathrm{C}, 10 \mathrm{mmHg}\right)$. The crude solid was purified by recrystallization by dissolving in boiling EtOAc $(10 \mathrm{~mL})$ and cooling the solution to r.t. and then to $-20{ }^{\circ} \mathrm{C}$ in a freezer. The $1^{\text {st }}$ crop was collected by filtration, and the mother liquor was concentrated and recrystallized (boiling EtOAc, $5 \mathrm{~mL}$ ). After cooling at $-20^{\circ} \mathrm{C}$ and filtration, a combined yield of $1.6 \mathrm{~g}(83 \%)$ of $\mathbf{1 b}$ was obtained; white solid; $\mathrm{mp} 114-115^{\circ} \mathrm{C}$ (sealed tube); $R_{f}=0.30$ (hexanes/EtOAc, 4:1) [UV].

IR (neat): 3271 (w), 3027 (w), 2957 (w), 2837 (w), 1599 (m), 1581 (w), 1496 (s), 1464 (w), 1448 (w), 1433 (w), 1399 (m), 1327 (m), 1304 (m), 1290 (m), 1215 (m), 1185 (w), 1159 (s), $1092(\mathrm{~m}), 1038(\mathrm{~m}), 968(\mathrm{~m}), 945(\mathrm{w}), 899(\mathrm{~m}), 814(\mathrm{~m}), 753(\mathrm{~m}), 731(\mathrm{~m}), 693(\mathrm{~m}), 665$ (m), $596(\mathrm{w}), 550(\mathrm{~m}) \mathrm{cm}^{-1}$.

${ }^{1} \mathrm{H} \mathrm{NMR}\left(500 \mathrm{MHz}, \mathrm{CDCl}_{3}\right): \delta=7.58(\mathrm{~d}, J=8.2 \mathrm{~Hz}, 2 \mathrm{H}, \mathrm{HC}(16)), 7.31-7.27(\mathrm{~m}, 4 \mathrm{H}$, $\mathrm{HC}(11,12)), 7.24-7.20(\mathrm{~m}, 3 \mathrm{H}, \mathrm{HC}(17,13)), 7.19(\mathrm{~d}, J=8.7 \mathrm{~Hz}, 1 \mathrm{H}, \mathrm{HC}(3)), 6.72$ (dd, $J=$ 8.7, $3.0 \mathrm{~Hz}, 1 \mathrm{H}, \mathrm{HC}(2)), 6.69$ (d, $J=2.9 \mathrm{~Hz}, 1 \mathrm{H}, \mathrm{HC}(6)), 6.31$ (s, $1 \mathrm{H}, \mathrm{HN}(14)), 6.22$ (dt, $J$ = 15.8, $1.7 \mathrm{~Hz}, 1 \mathrm{H}, \mathrm{HC}(9)), 6.06$ (dt, $J=15.9,6.5 \mathrm{~Hz}, 1 \mathrm{H}, \mathrm{HC}(8)), 3.77$ (s, $3 \mathrm{H}, \mathrm{HC}(20)$ ), 3.17 (dd, $J=6.5,1.6 \mathrm{~Hz}, 2 \mathrm{H}, \mathrm{HC}(7)), 2.39$ (s, $3 \mathrm{H}, \mathrm{HC}(19))$.

${ }^{13} \mathrm{C}$ NMR (126 MHz, $\mathrm{CDCl}_{3}$ ): $\delta=158.7$ (C1), 143.9 (C18), 137.02 (C4), 136.99 (C5), 132.0 (C9), 129.8 (C17), 128.8 (C12), 128.5 (C3), 127.73 (C13), 127.69 (C10 or C15), 127.5 (C16), 127.4 (C10 or C15), 127.3 (C8), 126.5 (C11), 116.0 (C6), 112.6 (C2), 55.6 (C20), 35.4 (C7), 21.8 (C19).

MS (ESI): $m / z(\%)=239(100)[\mathrm{M}-\mathrm{Ts}+\mathrm{H}]^{+}, 240(18), 394(45)[\mathrm{M}+\mathrm{H}]^{+}, 395(12), 411$ (10). 
HRMS (ESI): $m / z$ [M + H] $]^{+}$calcd for $\mathrm{C}_{23} \mathrm{H}_{24} \mathrm{NO}_{3} \mathrm{~S}: 394.1471$; found: 394.1479 .

Anal. Calcd for $\mathrm{C}_{23} \mathrm{H}_{23} \mathrm{NO}_{3} \mathrm{~S}$ (393.50): C, 70.20; H, 5.89; N, 3.56. Found: C, 69.85; H, $5.81 ; \mathrm{N}, 3.59$.

\section{(E)-1-Cinnamylnaphthalen-2-amine $(5 c)^{4 a}$}

To a 50-mL Schlenk flask equipped with a magnetic stir bar were added $\mathrm{N}$-(1-

phenylallyl)naphthalen-2-amine (3c) (259 mg, $1.0 \mathrm{mmol}$ ), TsOH$\cdot \mathrm{H}_{2} \mathrm{O}$ (39 mg, $0.2 \mathrm{mmol}, 0.2$ equiv), and a mixed solution of $\mathrm{MeCN}(10 \mathrm{~mL})$ and $\mathrm{H}_{2} \mathrm{O}(1 \mathrm{~mL})$. The solution was heated to $65^{\circ} \mathrm{C}$ in an oil bath for $6 \mathrm{~h}$. The solution was cooled to r.t. at which point a solid started to form. To this suspension were added $2 \mathrm{M} \mathrm{NaOH}(10 \mathrm{~mL})$ and $\mathrm{Et}_{2} \mathrm{O}(20 \mathrm{~mL})$. The organic layer was separated, washed with brine $(10 \mathrm{~mL})$, dried over $\mathrm{MgSO}_{4}$, and then concentrated under reduced pressure $\left(30^{\circ} \mathrm{C}, 10 \mathrm{mmHg}\right)$. Purification of the residue by silica gel flash chromatography $(16 \times 2 \mathrm{~cm} ; 300 \mathrm{~mL}$ of $95: 5$ hexane/EtOAc, $200 \mathrm{~mL}$ of 90:10 hexane/ EtOAc, $250 \mathrm{~mL}$ of 50:50 hexane/EtOAc) afforded $210 \mathrm{mg}(81 \%)$ of a brown liquid. Crystallization of the brown liquid was achieved by dissolving in boiling $\mathrm{Et}_{2} \mathrm{O}(0.5 \mathrm{~mL})$, followed by slow addition of pentane $(2 \mathrm{~mL})$. The solution was cooled to r.t. and then to $-20{ }^{\circ} \mathrm{C}$ in a freezer. Filtration over glass wool afforded $192 \mathrm{mg}(74 \%)$ of $\mathbf{5 c}$; white crystals; mp 66-68 ${ }^{\circ} \mathrm{C}$ (sealed tube); $R_{f}=0.40$ (hexanes/EtOAc, 4:1) [UV].

IR (neat): 3023 (w), 1622 (s), 1600 (m), 1496 (m), 1473 (m), 1446 (m), 1435 (m), 1393 (m), $1356(\mathrm{w}), 1282(\mathrm{~m}), 1259(\mathrm{~m}), 1222(\mathrm{w}), 1164(\mathrm{w}), 964(\mathrm{~s}), 908(\mathrm{~m}), 857(\mathrm{w}), 811(\mathrm{~s}), 782$ (m), $731(\mathrm{~s}), 691(\mathrm{~s}), 672(\mathrm{~m}), 648(\mathrm{~m}), 617(\mathrm{~m}), 599(\mathrm{~m}), 586(\mathrm{~m}), 545(\mathrm{~m}), 518(\mathrm{~m}), 500$ (m), $477(\mathrm{~m}) \mathrm{cm}^{-1}$.

${ }^{1} \mathrm{H}$ NMR $\left(500 \mathrm{MHz}, \mathrm{CDCl}_{3}\right): \delta=7.88(\mathrm{~d}, J=8.6 \mathrm{~Hz}, 1 \mathrm{H}, \mathrm{HC}(10)), 7.75(\mathrm{dt}, J=8.1,0.7$ $\mathrm{Hz}, 1 \mathrm{H}, \mathrm{HC}(13)), 7.64$ (d, $J=8.7 \mathrm{~Hz}, 1 \mathrm{H}, \mathrm{HC}(2)), 7.45$ (ddd, $J=8.5,6.8,1.4 \mathrm{~Hz}, 1 \mathrm{H}$, $\mathrm{HC}(11)), 7.28$ (tt, $J=5.4,1.5 \mathrm{~Hz}, 3 \mathrm{H}, \mathrm{HC}(12,15)), 7.26-7.22$ (m, $2 \mathrm{H}, \mathrm{HC}(16)), 7.17$ (tt, $J=$ 7.2, 2.2 Hz, $1 \mathrm{H}, \mathrm{HC}(17)), 6.99$ (d, $J=8.7 \mathrm{~Hz}, 1 \mathrm{H}, \mathrm{HC}(3)), 6.44-6.34$ (m, $2 \mathrm{H}, \mathrm{HC}(8,9))$, $3.86(\mathrm{~d}, J=4.5 \mathrm{~Hz}, 4 \mathrm{H}, \mathrm{HC}(7)$ and $\mathrm{HN}(18))$.

${ }^{13} \mathrm{C}$ NMR (126 MHz, $\mathrm{CDCl}_{3}$ ): $\delta$ = 142.3 (C4), 137.4 (C14), 133.6 (C6), 130.7 (C9), 128.9 (C13), 128.8 (C1), 128.7 (C16), 128.3 (C2), 127.4 (C17), 127.3 (C8), 126.8 (C11), 126.3 (C12), 122.5 (C10), 122.4 (C15), 119.1 (C3), 114.7 (C5), 29.9 (C7).

MS (ESI): $m / z(\%)=156(100)[\mathrm{M}-\text { styrene }+\mathrm{H}]^{+}, 157(11), 260(43)[\mathrm{M}+\mathrm{H}]^{+}, 261(10)$.

HRMS (ESI): $m / z$ [M $+\mathrm{H}]^{+}$calcd for $\mathrm{C}_{19} \mathrm{H}_{18} \mathrm{~N}$ : 260.1439; found: 260.1441 .

Anal. Calcd for $\mathrm{C}_{19} \mathrm{H}_{17} \mathrm{~N}$ (259.35): C, 87.99; H, 6.61; N, 5.40. Found: C, 87.76; H, 6.44; N, 5.50 .

\section{(E)-N-(1-Cinnamyl-2-naphthyl)-4-methylbenzenesulfonamide (1c) ${ }^{\mathbf{1 1}}$}

To a flame-dried, 50-mL Schlenk flask equipped with a magnetic stir bar, purged with a positive pressure of argon, were added aniline $5 \mathbf{c}(1.43 \mathrm{~g}, 5.5 \mathrm{mmol})$ and $\mathrm{CH}_{2} \mathrm{Cl}_{2}(17 \mathrm{~mL}$, $0.3 \mathrm{M})$. The solution was cooled to $0{ }^{\circ} \mathrm{C}$ and pyridine $(2.2 \mathrm{~mL}, 27.5 \mathrm{mmol}, 5.0$ equiv) and $\mathrm{TsCl}(1.57 \mathrm{~g}, 8.25 \mathrm{mmol}, 1.5$ equiv) were added. The reaction mixture was warmed to r.t. 
and stirred for $12 \mathrm{~h}$. Then, brine $(30 \mathrm{~mL})$ was added and the solution was extracted with $\mathrm{CH}_{2} \mathrm{Cl}_{2}(3 \times 30 \mathrm{~mL})$. The organic layers were combined, dried over $\mathrm{MgSO}_{4}$, filtered, and concentrated under reduced pressure $\left(30^{\circ} \mathrm{C}, 10 \mathrm{mmHg}\right)$ to afford a dark brown oil. The crude product was dissolved in $\mathrm{Et}_{2} \mathrm{O}$ and a yellow solid precipitated immediately. Recrystallization was undertaken by dissolving the yellow solid in boiling EtOAc $(10 \mathrm{~mL})$ and then cooling the solution to r.t. and subsequently to $-20{ }^{\circ} \mathrm{C}$ in a freezer. The $1^{\text {st }}$ crop was collected by filtration, and the mother liquor was concentrated and recrystallized (boiling EtOAc, $5 \mathrm{~mL})$. After cooling at $-20^{\circ} \mathrm{C}$ and filtration, a combined yield of $1.9 \mathrm{~g}(83 \%)$ of 1c was obtained; yellow solid; $\mathrm{mp} 148-149{ }^{\circ} \mathrm{C}$ (sealed tube); $R_{f}=0.38$ (hexanes/EtOAc, $4: 1$ ) [UV].

IR (neat): 3283 (w), 1598 (m), 1512 (w), 1496 (w), 1468 (w), 1447 (w), 1407 (m), 1367 (m), 1320 (m), 1304 (m), 1234 (w), 1185 (w), 1159 (s), 1092 (m), 1067 (w), 1019 (w), 966 (m), $907(\mathrm{~m}), 864(\mathrm{w}), 847(\mathrm{w}), 813(\mathrm{~m}), 763(\mathrm{~m}), 733$ (s), $706(\mathrm{~m}), 691(\mathrm{~m}), 669$ (s), $598(\mathrm{~m})$, $552(\mathrm{~s}), 532(\mathrm{~m}), 494(\mathrm{w}) \mathrm{cm}^{-1}$.

${ }^{1} \mathrm{H}$ NMR (500 MHz, $\left.\mathrm{CDCl}_{3}\right): \delta=7.92(\mathrm{~d}, J=8.5 \mathrm{~Hz}, 1 \mathrm{H}, \mathrm{HC}(10)), 7.84(\mathrm{~d}, J=8.1 \mathrm{~Hz}, 1$ $\mathrm{H}, \mathrm{HC}(2)), 7.75$ (d, $J=8.8 \mathrm{~Hz}, 1 \mathrm{H}, \mathrm{HC}(13)), 7.66$ (d, $J=8.8 \mathrm{~Hz}, 1 \mathrm{H}, \mathrm{HC}(12)), 7.61$ (d, $J=$ $8.1 \mathrm{~Hz}, 2 \mathrm{H}, \mathrm{HC}(20)), 7.53-7.42$ (m, $2 \mathrm{H}, \mathrm{HC}(11,3)), 7.24(\mathrm{dd}, J=7.2,1.2 \mathrm{~Hz}, 2 \mathrm{H}$, HC(16)), 7.22-7.17 (m, 3 H, HC(15,17)), 7.13 (d, J= 7.9 Hz, 2 H, HC(21)), 6.64 (s, 1 H, $\mathrm{HN}(18)), 6.19(\mathrm{dtd}, J=16.1,5.1,1.1 \mathrm{~Hz}, 1 \mathrm{H}, \mathrm{HC}(8)), 6.13(\mathrm{~d}, J=16.1 \mathrm{~Hz}, 1 \mathrm{H}, \mathrm{HC}(9))$, $3.65(\mathrm{dd}, J=5.4,1.3 \mathrm{~Hz}, 2 \mathrm{H}, \mathrm{HC}(7)), 2.31$ (s, $3 \mathrm{H}, \mathrm{HC}(23))$.

${ }^{13} \mathrm{C}$ NMR (126 MHz, $\left.\mathrm{CDCl}_{3}\right): \delta=144.1$ (C22), 136.9 (C14), 136.8 (C19), 132.7 (C6), 132.6 (C1), 132.4 (C5), 131.6 (C9), 129.9 (C21), 129.8 (C4), 128.9 (C2), 128.7 (C16), 128.4 (C13), 127.7 (C17), 127.4 (C20), 127.0 (C11), 126.8 (C8), 126.4 (C15), 125.8 (C3), 124.5 (C10), 123.6 (C12), 30.0 (C7), 21.8 (C23).

MS (ESI): $m / z(\%)=258(22), 259(100)[\mathrm{M}-\mathrm{Ts}+\mathrm{H}]^{+}, 260(18), 436(25)[\mathrm{M}+\mathrm{Na}]^{+}$. HRMS (ESI): $m / z[\mathrm{M}+\mathrm{Na}]^{+}$calcd for $\mathrm{C}_{26} \mathrm{H}_{23} \mathrm{NO}_{2} \mathrm{SNa}$ : 436.1342; found: 436.1347 .

Anal. Calcd for $\mathrm{C}_{26} \mathrm{H}_{23} \mathrm{NO}_{2} \mathrm{~S}$ (413.53): C, 75.52; H, 5.61; N, 3.39. Found: C, 75.19; H, $5.63 ; \mathrm{N}, 3.51$.

\section{N-(1-Isopropyl-2-propenyl)aniline (3d) (Scheme 5)}

By adopting the described procedure, ${ }^{8}$ to an oven-dried, $100-\mathrm{mL}$, round-bottomed flask equipped with a magnetic stir bar were added imine $2 \mathbf{d}^{5 \mathrm{~d}}(736 \mathrm{mg}, 5 \mathrm{mmol})$ and toluene (30 $\mathrm{mL}, 0.17 \mathrm{M})$. After a condenser was installed, the flask was purged with argon and 1(trimethylsilyl)benzotriazole (BtTMS; $957 \mathrm{mg}, 5 \mathrm{mmol}, 1$ equiv) was added by syringe. After the reaction mixture was stirred for $30 \mathrm{~min}$ at r.t., it was cooled to $0{ }^{\circ} \mathrm{C}$ in an ice bath and a solution of vinylmagnesium chloride in THF (6.25 mL, 1.6 M, $10 \mathrm{mmol}, 2$ equiv) was added by syringe. To the resulting yellow turbid mixture was added $\mathrm{Et}_{2} \mathrm{O}(10 \mathrm{~mL})$. The reaction mixture was refluxed under heat (bath temperature $90^{\circ} \mathrm{C}$ ) for $20 \mathrm{~h}$. After the reaction mixture was cooled to r.t., it was quenched by pouring into ice-water $(30 \mathrm{~mL})$, and then the mixture was extracted with $\mathrm{Et}_{2} \mathrm{O}(3 \times 60 \mathrm{~mL})$. The organic layers were combined 
and washed with aqueous $2 \mathrm{M} \mathrm{NaOH}$ solution $(2 \times 20 \mathrm{~mL})$ and $\mathrm{H}_{2} \mathrm{O}(2 \times 30 \mathrm{~mL})$. The resulting organic layer was dried over $\mathrm{Na}_{2} \mathrm{SO}_{4}$, filtered, and concentrated under reduced pressure $\left(30^{\circ} \mathrm{C}, 10 \mathrm{mmHg}\right)$. Purification by silica gel flash chromatography $(40 \mathrm{~g}, 25 \mathrm{~mm}$ $\varnothing$; hexanes to hexanes/EtOAc, 19:1) afforded $595 \mathrm{mg}$ (68\%) of $\mathbf{3 d}$ as a pale yellow oil. An analytically pure sample was obtained by Kugelrohr distillation $\left(60^{\circ} \mathrm{C} / 0.01 \mathrm{mmHg}\right)$, affording $550 \mathrm{mg}(63 \%)$ of $\mathbf{3 d}$ as a colorless oil; bp $60^{\circ} \mathrm{C} / 0.01 \mathrm{mmHg} ; R_{f}=0.58$ (hexanes/ EtOAc, 4:1) [UV]. The spectroscopic data matched those reported previously. ${ }^{19}$

${ }^{1} \mathrm{H} \mathrm{NMR}\left(500 \mathrm{MHz}, \mathrm{CDCl}_{3}\right): \delta=7.18(\mathrm{t}, J=8.0 \mathrm{~Hz}, 2 \mathrm{H}, \mathrm{HC}(3)), 6.69(\mathrm{t}, J=7.5 \mathrm{~Hz}, 1 \mathrm{H}$, $\mathrm{HC}(4)), 6.63$ (d, $J=8.5 \mathrm{~Hz}, 2 \mathrm{H}, \mathrm{HC}(2)), 5.76$ (ddd, $J=17.0,10.5,6.0 \mathrm{~Hz}, 1 \mathrm{H}, \mathrm{HC}(6)$ ), 5.27-5.16 (m, $2 \mathrm{H}, \mathrm{HC}(7)), 3.75-3.63$ (br s + m, 2 H, HN + HC(5)), 1.90 (dq, J= 13.5, 6.5 $\mathrm{Hz}, 1 \mathrm{H}, \mathrm{HC}(8)), 1.01$ (dd, $J=15.0,7.0 \mathrm{~Hz}, 6 \mathrm{H}, \mathrm{HC}(9)$ ).

${ }^{13} \mathrm{C}$ NMR (126 MHz, $\left.\mathrm{CDCl}_{3}\right): \delta=147.8(\mathrm{C} 1), 137.9(\mathrm{C} 6), 129.1(\mathrm{C} 3), 117.0(\mathrm{C} 4), 116.0$ (C7), 113.3 (C2), 61.4 (C5), 32.4 (C8), 18.8 (C9), 18.5 (C9).

MS (ESI): $m / z(\%)=94$ (20), 97 (22), 98 (15), 106 (14), 114 (56), 118 (28), 138 (16), 140 (13), 142 (26), 148 (34), 150 (28), 176 (78) [M + H], 188 (50), 202 (43), 219 (100), 220 (17), 230 (16), 235 (69), 248 (16), 251 (19), 258 (25), 262 (14), 274 (40).

HRMS (ESI): $m / z[\mathrm{M}+\mathrm{H}]^{+}$calcd for $\mathrm{C}_{12} \mathrm{H}_{18} \mathrm{~N}$ : 176.1439; found: 176.1440 .

\section{(E)-2-(4-Methyl-2-pentenyl)aniline (5d)}

By adopting the described procedure, ${ }^{7,11}$ to an oven-dried, $38-\mathrm{mL}$ pressure tube with a side arm in the neck, equipped with a magnetic stir bar, were added allylaniline $\mathbf{3 d}$ (350 $\mathrm{mg}, 2.0$ $\mathrm{mmol}$ ) and xylenes $(4 \mathrm{~mL}, 0.5 \mathrm{M})$. The tube was capped with a septum and purged with a positive pressure of argon. The solution was cooled to $-40{ }^{\circ} \mathrm{C}$ in a MeCN/dry ice bath under positive argon pressure. To the tube was added $\mathrm{BF}_{3} \cdot \mathrm{OEt}_{2}(48 \%$ solution, $0.62 \mathrm{~mL}, 2.4 \mathrm{mmol}$, 1.2 equiv). The reaction mixture was warmed to r.t. and the septum was replaced with a pressure screw cap under a positive stream of argon. Then, the mixture was heated to $180^{\circ} \mathrm{C}$ and stirred for $17 \mathrm{~h}$. After completion, the reaction mixture was cooled to $0{ }^{\circ} \mathrm{C}$ with continuous vigorous stirring and quenched with $2 \mathrm{M} \mathrm{NaOH}(15 \mathrm{~mL})$ at $0{ }^{\circ} \mathrm{C}$. The resulting biphasic layer was extracted with $\mathrm{Et}_{2} \mathrm{O}(3 \times 15 \mathrm{~mL})$. The organic layers were combined, filtered over Celite, and concentrated under reduced pressure $\left(30^{\circ} \mathrm{C}, 10 \mathrm{mmHg}\right)$. The crude solid was purified by silica gel flash chromatography (30 g, $20 \mathrm{~mm} \varnothing$; hexanes to hexanes/ EtOAc, 6:1) to afford $199 \mathrm{mg}(57 \%)$ of $\mathbf{5 d}$ as a yellow oil. An analytically pure sample was obtained by Kugelrohr distillation $\left(80^{\circ} \mathrm{C} / 0.01 \mathrm{mmHg}\right)$, affording $179 \mathrm{mg}(51 \%)$ of $\mathbf{5 d}$ as a colorless oil; bp $80{ }^{\circ} \mathrm{C} / 0.01 \mathrm{mmHg} ; R_{f}=0.43$ (hexanes/EtOAc, 4:1) [UV].

${ }^{1} \mathrm{H}$ NMR (500 MHz, $\mathrm{CDCl}_{3}$ ): $\delta=7.14-7.07(\mathrm{~m}, 2 \mathrm{H}, \mathrm{HC}(3,5)), 6.80$ (tt, $J=7.5,1.5 \mathrm{~Hz}, 1$ H, HC(4)), 6.72 (dd, $J=7.5,1.5 \mathrm{~Hz}, 1 \mathrm{H}, \mathrm{HC}(6)), 5.63-5.50$ (m, $2 \mathrm{H}, \mathrm{HC}(8,9)), 3.75$ (br s, 2 $\mathrm{H}, \mathrm{H}_{2} \mathrm{~N}$ ), 3.30 (d, $\left.J=5.5 \mathrm{~Hz}, 2 \mathrm{H}, \mathrm{HC}(7)\right), 2.35$ (hept d, $\left.J=6.5,2.0 \mathrm{~Hz}, 1 \mathrm{H}, \mathrm{HC}(10)\right), 1.04$ (dd, $J=7.0,2.0 \mathrm{~Hz}, 6 \mathrm{H}, \mathrm{HC}(11)$ ).

${ }^{13} \mathrm{C}$ NMR (126 MHz, $\mathrm{CDCl}_{3}$ ): $\delta$ = 144.9 (C1), 139.5 (C9), 129.9 (C5), 127.3 (C3), 125.1 (C2), 124.3 (C8), 118.7 (C4), 115.7 (C6), 35.4 (C7), 31.0 (C10), 22.5 (C11). 
MS (ESI): $m / z(\%)=106(100), 114(27), 176(13)[\mathrm{M}+\mathrm{H}]^{+}, 177(13)$.

HRMS (ESI): $m / z$ [M $+\mathrm{H}]^{+}$calcd for $\mathrm{C}_{12} \mathrm{H}_{18} \mathrm{~N}$ : 176.1439; found: 176.1443 .

Anal. Calcd for $\mathrm{C}_{12} \mathrm{H}_{17} \mathrm{~N}$ (175.27): C, 82.23; H, 9.78; N, 7.99. Found: C, 82.25; H, 10.00; N, 7.80 .

\section{(E)-4-Methyl-N-[2-(4-methyl-2-pentenyl)phenyl]benzenesulfonamide (1d) ${ }^{11}$}

To an oven-dried, $50-\mathrm{mL}$, round-bottomed flask equipped with a magnetic stir bar were added aniline $5 \mathbf{d}(876 \mathrm{mg}, 5.0 \mathrm{mmol})$ and $\mathrm{CH}_{2}-\mathrm{Cl}_{2}(10 \mathrm{~mL}, 0.5 \mathrm{M})$ under positive argon pressure. The solution was cooled to $0{ }^{\circ} \mathrm{C}$ and pyridine $(1.21 \mathrm{~mL}, 15.0 \mathrm{mmol}, 3.0$ equiv) and $\mathrm{TsCl}$ (1.05 g, $5.5 \mathrm{mmol}, 1.1$ equiv) were added. The reaction mixture was warmed to r.t. and stirred for $24 \mathrm{~h}$. To quench the reaction, $\mathrm{H}_{2} \mathrm{O}(20 \mathrm{~mL})$ was added and then the solution was extracted with $\mathrm{CH}_{2} \mathrm{Cl}_{2}(3 \times 20 \mathrm{~mL})$. The organic layers were combined, dried over $\mathrm{Na}_{2} \mathrm{SO}_{4}$, filtered, and concentrated under reduced pressure $\left(30^{\circ} \mathrm{C}, 10 \mathrm{mmHg}\right)$. The crude solid was purified by silica gel flash chromatography $(40 \mathrm{~g}, 25 \mathrm{~mm} \varnothing$; hexanes to hexanes/EtOAc, 6:1) to afford $1.48 \mathrm{~g}(90 \%)$ of $\mathbf{1 d}$ as a yellow solid. An analytically pure sample was obtained by recrystallization (boiling pentane, $50 \mathrm{~mL}$ ). The solution was cooled to r.t. and then to $-20^{\circ} \mathrm{C}$ in a freezer. The $1^{\text {st }}$ crop was collected by filtration, and the mother liquor was concentrated and recrystallized (boiling pentane, $10 \mathrm{~mL}$ ). After cooling at $-20{ }^{\circ} \mathrm{C}$ and filtration, a combined yield of $1.34 \mathrm{~g}(82 \%)$ of $\mathbf{1 d}$ was obtained; white crystalline solid; $\mathrm{mp}$ $128-129{ }^{\circ} \mathrm{C}$ (sealed tube); $R_{f}=0.32$ (hexanes/EtOAc, 4:1) [UV].

IR (neat): 3286 (m), 2963 (w), 1489 (m), 1458 (w), 1391 (m), 1334 (s), 1307 (w), $1291(\mathrm{w})$, 1275 (w), 1187 (w), 1166 (s), 1119 (w), 1090 (s), 1045 (w), 1021 (w), 969 (m), 901 (m), 834 (w), $810(\mathrm{~m}), 762(\mathrm{~s}) \mathrm{cm}^{-1}$.

${ }^{1} \mathrm{H} \mathrm{NMR}\left(500 \mathrm{MHz}, \mathrm{CDCl}_{3}\right): \delta=7.60(\mathrm{~d}, J=8.0 \mathrm{~Hz}, 2 \mathrm{H}, \mathrm{HC}(8)), 7.43(\mathrm{~d}, J=8.0 \mathrm{~Hz}, 1 \mathrm{H}$, $\mathrm{HC}(6)), 7.22$ (d, $J=8.0 \mathrm{~Hz}, 2 \mathrm{H}, \mathrm{HC}(9)), 7.19$ (t, $J=7.5 \mathrm{~Hz}, 1 \mathrm{H}, \mathrm{HC}(5)), 7.09$ (t, $J=7.5$

$\mathrm{Hz}, 1 \mathrm{H}, \mathrm{HC}(4)), 7.04$ (d, $J=7.5 \mathrm{~Hz}, 1 \mathrm{H}, \mathrm{HC}(3)), 6.69$ (s, $1 \mathrm{H}, \mathrm{HN}$ ), 5.44 (dd, $J=15.5,6.5$ $\mathrm{Hz}, 1 \mathrm{H}, \mathrm{HC}(14)), 5.31$ (dt, $J=15.5,6.0 \mathrm{~Hz}, 1 \mathrm{H}, \mathrm{HC}(13)), 2.93$ (d, $J=6.0 \mathrm{~Hz}, 2 \mathrm{H}$, $\mathrm{HC}(12)), 2.39$ (s, $3 \mathrm{H}, \mathrm{HC}(11)), 2.34-2.22$ (m, $1 \mathrm{H}, \mathrm{HC}(15)), 0.99$ (d, J= $7.0 \mathrm{~Hz}, 6 \mathrm{H}$, $\mathrm{HC}(16))$.

${ }^{13} \mathrm{C}$ NMR (126 MHz, $\mathrm{CDCl}_{3}$ ): $\delta=143.7$ (C10), 140.7 (C14), 136.9 (C7), 135.3 (C1), 132.2 (C2), 130.3 (C3), 129.6 (C9), 127.6 (C5), 127.0 (C8), 125.9 (C4), 124.0 (C13), 123.9 (C6), 35.5 (C12), 31.0 (C15), 22.3 (C16), 21.5 (C11).

MS (ESI): $m / z(\%)=118$ (52), 132 (22), 146 (13), 160 (39), 174 (17), 175 (100), 176 (14), $330(36)[\mathrm{M}+\mathrm{H}]^{+}, 352(42)$.

HRMS (ESI): $\mathrm{m} / z$ [M + H] $]^{+}$calcd for $\mathrm{C}_{19} \mathrm{H}_{24} \mathrm{NO}_{2} \mathrm{~S}: 330.1528$; found: 330.1526 .

Anal. Calcd for $\mathrm{C}_{19} \mathrm{H}_{23} \mathrm{NO}_{2} \mathrm{~S}$ (329.46): C, 69.27; H, 7.04; N, 4.25. Found: C, 69.42; H, $7.23 ; \mathrm{N}, 4.26$. 


\section{Olefin Cross-Metathesis (Table 4)}

\section{(E)-4-Methyl-N-[2-(3-phenyl-2-propenyl)phenyl]benzenesulfonamide (1e)}

By adopting the described procedure, ${ }^{21}$ an oven-dried, 50-mL Schlenk flask equipped with a magnetic stir bar was introduced into a glovebox. Grubbs $1^{\text {st }}$ generation indenylidene catalyst (CAS no. 250220-36-1; $111 \mathrm{mg}, 0.12 \mathrm{mmol}, 0.03$ equiv) was loaded into the flask, which was sealed with a septum and removed from the glovebox. The Schlenk flask was hooked up to a Schlenk manifold and was purged thoroughly with argon. To a separate 50$\mathrm{mL}$, round-bottomed flask were added sulfonamide $\mathbf{6}^{11}$ (1.15 g, $4.0 \mathrm{mmol}, 1$ equiv), styrene (4.60 mL, $40.0 \mathrm{mmol}, 10$ equiv), and $\mathrm{CH}_{2} \mathrm{Cl}_{2}$ ( $20 \mathrm{~mL}, 0.2 \mathrm{M}$, degassed with argon); then, the flask was sealed with a septum. The mixture turned into a solution while being degassed for an additional 30 min with argon (needle and a bubbler outlet attached). The premixed, degassed solution of the two olefin substrates was transferred to the Schlenk flask by syringe. The top area of the Schlenk flask, including the nee dle-punctured septum, was thoroughly sealed with Parafilm. The black mixture was stirred for $48 \mathrm{~h}$ at r.t. The mixture was filtered through a plug of silica gel, then rinsed with $\mathrm{CH}_{2} \mathrm{Cl}_{2}(2 \times 25 \mathrm{~mL})$ and EtOAc (2 $\times 25 \mathrm{~mL}$ ) to afford a dark solution. The filtrate was concentrated under reduced pressure $\left(30{ }^{\circ} \mathrm{C}, 10 \mathrm{mmHg}\right)$ to afford a black solid. Purification by silica gel flash chromatography (40 g, $25 \mathrm{~mm} \varnothing$; hexanes to hexanes/EtOAc, 6:1) afforded $685 \mathrm{mg}(47 \%)$ of $1 \mathrm{e}$ as a white solid. Recrystallization of the solid with hot pentane $\left(36^{\circ} \mathrm{C}, 30 \mathrm{~mL}\right)$ afforded $630 \mathrm{mg}(43 \%)$ of $1 \mathrm{e}$ as white crystals; $\mathrm{mp} 159-160^{\circ} \mathrm{C}$ (sealed tube). The spectroscopic data matched those reported previously. ${ }^{22}$

IR (neat): 3280 (m), 1492 (m), 1448 (w), 1403 (m), 1333 (s), 1305 (w), $1291(\mathrm{w}), 1278$ (w), 1184 (w), 1165 (s), 1119 (w), 1090 (s), 1053 (w), 1040 (w), 1019 (w), 969 (m), 954 (w), 907 (s), $810(\mathrm{~m}), 756(\mathrm{~m}) \mathrm{cm}^{-1}$.

${ }^{1} \mathrm{H} \mathrm{NMR}\left(500 \mathrm{MHz}, \mathrm{CDCl}_{3}\right): \delta=7.60(\mathrm{~d}, J=8.0 \mathrm{~Hz}, 2 \mathrm{H}, \mathrm{HC}(9)), 7.43(\mathrm{~d}, J=8.0 \mathrm{~Hz}, 1 \mathrm{H}$, $\mathrm{HC}(6)), 7.35-7.21$ (m, $6 \mathrm{H}, \mathrm{HC}(\operatorname{aryl})), 7.21-7.13$ (m, $4 \mathrm{H}, \mathrm{HC}(\operatorname{aryl})), 6.52$ (br s, $1 \mathrm{H}, \mathrm{HN}$ ), 6.29 (dt, $J=16.0,1.5 \mathrm{~Hz}, 1 \mathrm{H}, \mathrm{HC}(14)), 6.11$ (dt, $J=16.0,6.5 \mathrm{~Hz}, 1 \mathrm{H}, \mathrm{HC}(13)), 3.25$ (dd, $J$ $=6.5,1.5 \mathrm{~Hz}, 2 \mathrm{H}, \mathrm{HC}(12)), 2.39$ (s, $3 \mathrm{H}, \mathrm{HC}(11))$.

${ }^{13} \mathrm{C}$ NMR (126 MHz, $\left.\mathrm{CDCl}_{3}\right): \delta=143.8(\mathrm{C} 10), 136.6(\mathrm{C} 7), 136.6(\mathrm{C} 15), 134.9(\mathrm{C} 1), 132.2$ (C2), 131.9 (C14), 130.5 (C3), 129.6 (C9), 128.6 (C17), 127.8, 127.6, 127.2 (C8), 127.0 (C13), 126.2 (C16), 126.2, 124.2 (C6), 35.2 (C12), 21.5 (C11).

MS (ESI): $m / z(\%)=208(15), 209(100), 210$ (24), 364 (28) $[\mathrm{M}+\mathrm{H}]^{+}, 381$ (44), 382 (13), 386 (17).

HRMS (ESI): $\mathrm{m} / z$ [M + H] $]^{+}$calcd for $\mathrm{C}_{22} \mathrm{H}_{22} \mathrm{NO}_{2} \mathrm{~S}: 364.1371$; found: 364.1373 .

\section{(E)-N-\{2-[3-(4-Methoxyphenyl)-2-propenyl]phenyl\}-4-methyl-benzenesulfonamide (1f)}

By adopting the described procedure, ${ }^{21}$ an oven-dried, $25-\mathrm{mL}$ Schlenk flask equipped with a magnetic stir bar was introduced into a glove-box. Grubbs $1^{\text {st }}$ generation indenylidene catalyst (CAS no. 250220-36-1; $55 \mathrm{mg}, 0.06 \mathrm{mmol}, 0.03$ equiv) was loaded into the flask, which was sealed with a septum and removed from the glovebox. The Schlenk flask was 
hooked up to a Schlenk manifold and was purged thoroughly with argon. To a separate 25$\mathrm{mL}$, round-bottomed flask were added sulfonamide $\boldsymbol{6}^{11}$ (575 mg, $2.0 \mathrm{mmol}, 1$ equiv), 4vinylanisole (2.66 mL, $20.0 \mathrm{mmol}, 10$ equiv), and $\mathrm{CH}_{2} \mathrm{Cl}_{2}(10 \mathrm{~mL}, 0.2 \mathrm{M}$, degassed with argon); then, the flask was sealed with a septum. The mixture turned into a solution while being degassed for an additional 30 min with argon (needle and a bubbler outlet attached). The premixed, degassed solution of the two olefin substrates was transferred to the Schlenk flask by syringe. The top area of the Schlenk flask, including the needle-punctured septum, was thoroughly sealed with Parafilm. The black mixture was stirred for $48 \mathrm{~h}$ at r.t. The mixture was filtered through a plug of silica gel, then rinsed with $\mathrm{CH}_{2} \mathrm{Cl}_{2}(2 \times 15 \mathrm{~mL})$ and EtOAc $(2 \times 15 \mathrm{~mL})$ to afford a dark solution. The filtrate was concentrated under reduced pressure $\left(30^{\circ} \mathrm{C}, 10 \mathrm{mmHg}\right)$ to afford a black solid. Purification by silica gel flash chromatography (25 g, $20 \mathrm{~mm} \varnothing$; hexanes to hexanes/EtOAc, 6:1) afforded $425 \mathrm{mg}$ (54\%) of $\mathbf{1 f}$ as a white solid. Recrystallization of the solid with hot pentane $\left(36^{\circ} \mathrm{C}, 30 \mathrm{~mL}\right)$ afforded $396 \mathrm{mg}(50 \%)$ of $\mathbf{1 f}$ as white crystals; $\mathrm{mp} 117-118^{\circ} \mathrm{C}$ (sealed tube); $R_{f}=0.20$ (hexanes/EtOAc, 4:1) [UV].

IR (neat): 3271 (m), 3005 (w), 2965 (w), 1739 (w), 1606 (m), 1578 (w), 1510 (m), 1488 (m), 1469 (w), 1456 (w), 1437 (w), 1406 (m), 1332 (m), 1308 (w), 1292 (m), 1275 (w), 1251 (s), 1177 (w), 1160 (s), 1121 (w), 1109 (w), 1089 (m), 1066 (w), 1032 (m), 1018 (w), 969 (m), $908(\mathrm{~m}), 862(\mathrm{w}), 820(\mathrm{~m}), 812(\mathrm{~m}), 778(\mathrm{w}), 761(\mathrm{~m}), 754(\mathrm{~m}) \mathrm{cm}^{-1}$.

${ }^{1} \mathrm{H} \mathrm{NMR}\left(500 \mathrm{MHz}, \mathrm{CDCl}_{3}\right): \delta=7.58(\mathrm{~d}, J=8.5 \mathrm{~Hz}, 2 \mathrm{H}, \mathrm{HC}(8)), 7.42(\mathrm{~d}, J=8.0 \mathrm{~Hz}, 1 \mathrm{H}$, $\mathrm{HC}(6)), 7.25-7.13$ (m, $7 \mathrm{H}), 6.84$ (d, $J=8.5 \mathrm{~Hz}, 2 \mathrm{H}, \mathrm{HC}(17)), 6.56$ (br s, $1 \mathrm{H}, \mathrm{HN}$ ), 6.22 (d, $J=16.0 \mathrm{~Hz}, 1 \mathrm{H}, \mathrm{HC}(14)), 5.94$ (dt, $J=16.0,6.5 \mathrm{~Hz}, 1 \mathrm{H}, \mathrm{HC}(13)), 3.81$ (s, $3 \mathrm{H}, \mathrm{HC}(19)$ ), 3.19 (dd, $J=6.5,2.0 \mathrm{~Hz}, 2 \mathrm{H}, \mathrm{HC}(12)), 2.37$ (s, $3 \mathrm{H}, \mathrm{HC}(11)$ ).

${ }^{13} \mathrm{C}$ NMR (126 MHz, $\left.\mathrm{CDCl}_{3}\right): \delta=159.1$ (C18), 143.7 (C10), 136.6 (C7), $134.9(\mathrm{C} 1), 132.3$ (C2), 131.3 (C14), 130.5 (C3), 129.6 (C9), 129.4, 127.6, 127.4 (C8), 127.1 (C16), 126.1, 124.7 (C13), 124.0 (C6), 113.9 (C17), 55.3 (C19), 35.1 (C12), 21.5 (C11).

MS (ESI): $m / z(\%)=238(17), 239(100), 240$ (37), 297 (34), 394 (52) $[\mathrm{M}+\mathrm{H}]^{+}, 395$ (16), 411 (79), $412(25)$.

HRMS (ESI): $m / z$ [M $+\mathrm{H}]^{+}$calcd for $\mathrm{C}_{23} \mathrm{H}_{24} \mathrm{NO}_{3} \mathrm{~S}: 394.1477$; found: 394.1484 .

Anal. Calcd for $\mathrm{C}_{23} \mathrm{H}_{23} \mathrm{NO}_{3} \mathrm{~S}$ (393.50): C, 70.20; H, 5.89; N, 3.56. Found: C, 70.05; H, $5.81 ; \mathrm{N}, 3.55$.

\section{(E)- $N$-\{2-[3-(4-Bromophenyl)-2-propenyl]phenyl\}-4-methylbenzenesulfonamide (1g)}

By adopting the described procedure, ${ }^{21}$ an oven-dried, $25-\mathrm{mL}$ Schlenk flask equipped with a magnetic stir bar was introduced into a glove-box. Grubbs $1^{\text {st }}$ generation indenylidene catalyst (CAS no. 250220-36-1; $55 \mathrm{mg}, 0.06 \mathrm{mmol}, 0.03$ equiv) was loaded into the flask, which was sealed with a septum and removed from the glovebox. The Schlenk flask was hooked up to a Schlenk manifold and was purged thoroughly with argon. To a separate 25$\mathrm{mL}$, round-bottomed flask were added sulfonamide $\mathbf{6}^{11}$ (575 mg, $2.0 \mathrm{mmol}, 1$ equiv), 4bromostyrene (2.62 mL, $20.0 \mathrm{mmol}, 10$ equiv), and $\mathrm{CH}_{2} \mathrm{Cl}_{2}(10 \mathrm{~mL}, 0.2 \mathrm{M}$, degassed with 
argon); then, the flask was sealed with a septum. The mixture turned into a solution while being degassed for an additional $30 \mathrm{~min}$ with argon (needle and a bubbler outlet attached). The premixed, degassed solution of the two olefin substrates was transferred to the Schlenk flask by syringe. The top area of the Schlenk flask, including the needle-punctured septum, was thoroughly sealed with Parafilm. The black mixture was stirred for $48 \mathrm{~h}$ at r.t. The mixture was filtered through a plug of silica gel, then rinsed with $\mathrm{CH}_{2} \mathrm{Cl}_{2}(2 \times 15 \mathrm{~mL})$ and EtOAc $(2 \times 15 \mathrm{~mL})$ to afford a dark solution. The filtrate was concentrated under reduced pressure $\left(30{ }^{\circ} \mathrm{C}, 10 \mathrm{mmHg}\right)$ to afford a black solid. Purification by silica gel flash chromatography (25 g, $20 \mathrm{~mm} \varnothing$; hexanes to hexanes/EtOAc, 6:1) afforded $440 \mathrm{mg}(50 \%)$ of $\mathbf{1 g}$ as a white solid. Recrystallization of the solid with hot pentane $\left(36^{\circ} \mathrm{C}, 30 \mathrm{~mL}\right)$ afforded $404 \mathrm{mg}$ (46\%) of $\mathbf{1 g}$ as white crystals; mp $141-142{ }^{\circ} \mathrm{C}$ (sealed tube); $R_{f}=0.22$ (hexanes/EtOAc, 4:1) [UV].

${ }^{1} \mathrm{H}$ NMR (500 MHz, $\left.\mathrm{CDCl}_{3}\right): \delta=7.62(\mathrm{~d}, J=8.5 \mathrm{~Hz}, 2 \mathrm{H}, \mathrm{HC}(8)), 7.45(\mathrm{~d}, J=8.5 \mathrm{~Hz}, 2 \mathrm{H}$, $\mathrm{HC}(17)), 7.41$ (dd, $J=8.0,1.0 \mathrm{~Hz}, 1 \mathrm{H}, \mathrm{HC}(6)), 7.25$ (ddd, $J=8.5,6.0,3.0 \mathrm{~Hz}, 1 \mathrm{H}$, HC(aryl)), 7.22 (dd, J=8.0, 1.0 Hz, 2 H, HC(9)), 7.20-7.15 (m, 4 H, HC(aryl)), 6.5 (br s, 1 H, HN), 6.23 (d, $J=16.0 \mathrm{~Hz}, 1 \mathrm{H}, \mathrm{HC}(14)), 6.15$ (dt, $J=16.0,6.0 \mathrm{~Hz}, 1 \mathrm{H}, \mathrm{HC}(13)), 3.29$ (dd, $J=6.0,1.5 \mathrm{~Hz}, 2 \mathrm{H}, \mathrm{HC}(12)), 2.41$ (s, $3 \mathrm{H}, \mathrm{HC}(11))$.

${ }^{13} \mathrm{C}$ NMR (126 MHz, $\left.\mathrm{CDCl}_{3}\right): \delta=144.1$ (C10), 136.8 (C7), 135.8 (C15), 135.0 (C1), 132.5 (C2), 131.9 (C17), 130.9 (C14), 130.8 (C3), 129.9 (C9), 128.2 (C13), 128.1 (C5), 128.0 (C16), 127.4 (C8), 126.7 (C4), 124.6 (C6), 121.6 (C18), 35.3 (C12), 21.8 (C11).

MS (ESI): $m / z(\%)=440(95)[\mathrm{M}-\mathrm{H}]^{+}, 441(22), 442(100), 443$ (23), 456 (13).

HRMS (ESI): $\mathrm{m} / z[\mathrm{M}-\mathrm{H}]^{+}$calcd for $\mathrm{C}_{22} \mathrm{H}_{19} \mathrm{BrNO}_{2} \mathrm{~S}: 440.0320$; found: 440.0318 .

Anal. Calcd for $\mathrm{C}_{22} \mathrm{H}_{20} \mathrm{BrNO}_{2} \mathrm{~S}$ (442.37): C, 59.73; H, 4.56; N, 3.17. Found: C, 59.93; H, $4.39 ; \mathrm{N}, 3.13$.

\section{(E)-4-Methyl-N-[2-(4-phenyl-3-butenyl)phenyl]benzenesulfon-amide (1h)}

By adopting the described procedure, ${ }^{21}$ an oven-dried, 50-mL Schlenk flask equipped with a magnetic stir bar was introduced into a glovebox. Grubbs $1^{\text {st }}$ generation indenylidene catalyst (CAS no. 250220-36-1; $110 \mathrm{mg}, 0.12 \mathrm{mmol}, 0.03$ equiv) was loaded into the flask, which was sealed with a septum and removed from the glovebox. The Schlenk flask was hooked up to a Schlenk manifold and was purged thoroughly with argon. To a separate 25$\mathrm{mL}$, round-bottomed flask were added sulfonamide $7^{12}(1.21 \mathrm{~g}, 4.0 \mathrm{mmol}, 1$ equiv), styrene (4.60 mL, $40.0 \mathrm{mmol}, 10$ equiv), and $\mathrm{CH}_{2} \mathrm{Cl}_{2}$ (16 mL, $0.25 \mathrm{M}$, degassed with argon); then, the flask was sealed with a septum. The mixture turned into a solution while being degassed for an additional $30 \mathrm{~min}$ with argon (needle and a bubbler outlet attached). The premixed, degassed solution of the two olefin substrates was transferred to the Schlenk flask by syringe. The top area of the Schlenk flask, including the needle-punctured septum, was thoroughly sealed with Parafilm. The black mixture was stirred for $48 \mathrm{~h}$ at r.t. The mixture was filtered through a plug of silica gel, then rinsed with $\mathrm{CH}_{2} \mathrm{Cl}_{2}(2 \times 30 \mathrm{~mL})$ and EtOAc (2 $\times 30 \mathrm{~mL}$ ) to afford a dark solution. The filtrate was concentrated under reduced pressure $\left(30{ }^{\circ} \mathrm{C}, 10 \mathrm{mmHg}\right)$ to afford a black solid. Purification by silica gel flash chromatography 
(40 g, $25 \mathrm{~mm} \varnothing$; hexanes to hexanes/EtOAc, 6:1) afforded $740 \mathrm{mg}(49 \%)$ of $\mathbf{1 h}$ as a gray solid. Recrystallization of the solid (boiling EtOAc/pentane, 1:10; $20 \mathrm{~mL}$ ) afforded $649 \mathrm{mg}$ (43\%) of $\mathbf{1 h}$ as white crystals; mp $110-111^{\circ} \mathrm{C}$ (sealed tube); $R_{f}=0.25$ (hexanes/EtOAc, $4: 1)$ [UV].

IR (neat): 3222 (m), 1597 (w), 1493 (w), 1456 (w), 1448 (w), 1411 (w), 1324 (s), 1305 (w), 1294 (w), 1269 (w), 1185 (w), 1155 (s), 1120 (w), 1090 (s), 985 (w), 964 (m), 913 (m), 812 (m), $795(\mathrm{w}), 766(\mathrm{~s}) \mathrm{cm}^{-1}$.

${ }^{1} \mathrm{H} \mathrm{NMR}\left(500 \mathrm{MHz}, \mathrm{CDCl}_{3}\right): \delta=7.61(\mathrm{~d}, J=8.5 \mathrm{~Hz}, 2 \mathrm{H}, \mathrm{HC}(8)), 7.33-7.27(\mathrm{~m}, 5 \mathrm{H}$, HC(17,18,aryl)), 7.25-7.19 (m, 3 H, HC(aryl,9)), 7.17-7.11 (m, 3 H, HC(aryl)), 6.30 (d + br $\mathrm{s}, J=16.0 \mathrm{~Hz}, 2 \mathrm{H}, \mathrm{HC}(15)+\mathrm{HN}), 6.10(\mathrm{dt}, J=16.0,7.0 \mathrm{~Hz}, 1 \mathrm{H}, \mathrm{HC}(14)), 2.54(\mathrm{dd}, J=$ 9.0, 6.5 Hz, 2 H, HC(12)), 2.37 (s, 3 H, HC(11)), 2.30 (qd, J= 7.5, 7.0 Hz, 2 H, HC(13)).

${ }^{13} \mathrm{C}$ NMR (126 MHz, $\left.\mathrm{CDCl}_{3}\right): \delta=143.8$ (C10), 137.3 (C16), 136.7 (C7), 135.1 (C2), 134.0 (C1), 131.0 (C14), 129.9 (C3), 129.6 (C9), 128.9 (C15), 128.5 (C18), 127.2 (C8), 127.2, 127.1, 126.5, 126.0 (C17), 124.8 (C6), 33.2 (C13), 30.7 (C12), 21.5 (C11).

MS (ESI): $m / z(\%)=194$ (13), $222(22), 223(100), 224(16), 378(49)[\mathrm{M}+\mathrm{H}]^{+}, 379(13)$, 400 (32).

HRMS (ESI): $m / z[\mathrm{M}+\mathrm{H}]^{+}$calcd for $\mathrm{C}_{23} \mathrm{H}_{24} \mathrm{NO}_{2} \mathrm{~S}: 378.1528$; found: 378.1525 .

Anal. Calcd for $\mathrm{C}_{23} \mathrm{H}_{23} \mathrm{NO}_{2} \mathrm{~S}$ (377.50): C, 73.18; H, 6.14; N, 3.71. Found: C, 72.99; H, $6.29 ; \mathrm{N}, 3.62$.

\section{(E)-6-(2-Chlorophenyl)-4-hexenenitrile (11) (Scheme 9)}

To an oven-dried, 200-mL, round-bottomed flask equipped with a magnetic stir bar were added homoallylic alcohol $\mathbf{1 0}^{15}(787 \mathrm{mg}, 4.0 \mathrm{mmol})$ and $\mathrm{CH}_{2} \mathrm{Cl}_{2}(40 \mathrm{~mL}, 0.1 \mathrm{M})$, and then the flask was connected to an argon inlet. The solution was cooled to $0{ }^{\circ} \mathrm{C}$ in an ice bath, and $\mathrm{Et}_{3} \mathrm{~N}(1.95 \mathrm{~mL}, 14.0 \mathrm{mmol}, 3.5$ equiv) and $\mathrm{MsCl}(0.46 \mathrm{~mL}, 6.0 \mathrm{mmol}, 1.5$ equiv) were added by syringe. The reaction mixture was stirred for $1 \mathrm{~h}$ at $0{ }^{\circ} \mathrm{C}$. The reaction was quenched by adding $\mathrm{H}_{2} \mathrm{O}(20 \mathrm{~mL})$ and the resulting biphasic solution was separated. The aqueous layer was further extracted with $\mathrm{CH}_{2} \mathrm{Cl}_{2}(2 \times 30 \mathrm{~mL})$. The combined organic layer was dried over $\mathrm{Na}_{2} \mathrm{SO}_{4}$ and filtered over glass wool. The filtrate was concentrated under reduced pressure $\left(30{ }^{\circ} \mathrm{C}, 10 \mathrm{mmHg}\right)$ to afford a brown oil. Purification by silica gel plug filtration $(10 \mathrm{~g}, 30$ $\mathrm{mm} \varnothing$; hexanes/EtOAc, 7:3) afforded $1.04 \mathrm{~g} \mathrm{(95 \% )}$ of the mesylate as a pale yellow oil. (The mesylate intermediate slowly turned into a dark oil upon standing.) To an oven-dried, 100-mL, round-bottomed flask with a magnetic stir bar were added the mesylate $(1.04 \mathrm{~g}$, $3.79 \mathrm{mmol}$ ), DMF (30 mL, $0.125 \mathrm{M}$ ), and $\mathrm{NaCN}$ (557 mg, $11.4 \mathrm{mmol}, 3$ equiv). A condenser was installed on the flask, and connected to an argon inlet. The flask was purged with argon and the solution was stirred for $12 \mathrm{~h}$ at $40{ }^{\circ} \mathrm{C}$. Then, the flask was cooled to r.t. and the reaction was quenched by pouring into ice-water $(30 \mathrm{~mL})$. The aqueous layer was extracted with $\mathrm{CH}_{2} \mathrm{Cl}_{2}(3 \times 30 \mathrm{~mL})$, and each layer was washed with brine $(2 \times 20 \mathrm{~mL})$. The organic layers were combined, dried over $\mathrm{Na}_{2} \mathrm{SO}_{4}$, and concentrated under reduced pressure $\left(25^{\circ} \mathrm{C}, 10 \mathrm{mmHg}\right)$. Residual DMF was additionally removed by passage through a silica 
plug (10 g), rinsed with 4:1 hexanes/EtOAc. The collected organic layer was concentrated, and was purified by silica gel flash chromatography $(40 \mathrm{~g}, 25 \mathrm{~mm} \varnothing$; hexanes to hexanes/ EtOAc, 4:1) to afford $701 \mathrm{mg}$ (90\%) of $\mathbf{1 1}$ as a colorless oil; bp $80{ }^{\circ} \mathrm{C} / 0.1 \mathrm{mmHg} ; \boldsymbol{R}_{f}=0.53$ (hexanes/EtOAc, 4:1) [UV].

${ }^{1} \mathrm{H}$ NMR $\left(500 \mathrm{MHz}, \mathrm{CDCl}_{3}\right): \delta=7.39(\mathrm{dd}, J=8.0,1.5 \mathrm{~Hz}, 1 \mathrm{H}, \mathrm{HC}(9)), 7.28-7.16(\mathrm{~m}, 3 \mathrm{H}$, $\mathrm{HC}(\operatorname{aryl})), 5.79$ (dtd, $J=15.0,6.5,1.5 \mathrm{~Hz}, 1 \mathrm{H}, \mathrm{HC}(5)), 5.54$ (dddt, $J=15.0,6.5,5.0,1.5$ $\mathrm{Hz}, 1 \mathrm{H}, \mathrm{HC}(4)), 3.52$ (dd, $J=6.5,1.5 \mathrm{~Hz}, 2 \mathrm{H}, \mathrm{HC}(6)$ ), 2.47-2.36 (m, 4 H, HC(2,3)).

${ }^{13} \mathrm{C}$ NMR (126 MHz, $\left.\mathrm{CDCl}_{3}\right): \delta=137.6(\mathrm{C} 7), 133.9(\mathrm{C} 8), 130.6$ (C5), 130.3 (C12), 129.4 (C9), 127.8 (C4), 127.6 (C11), 126.9 (C10), 119.2 (C1), 36.3 (C6), 28.3 (C3), 17.5 (C2).

MS (EI): $m / z(\%)=89(11), 115$ (30), $116(25), 125(30), 128$ (25), 129 (100), 130 (26), 151 (64), 153 (20), 164 (14), 170 (50), 205 (45) [M] $]^{+}, 207$ (15).

HRMS (EI): $m / z$ [M] ${ }^{+}$calcd for $\mathrm{C}_{12} \mathrm{H}_{12} \mathrm{NCl}$ : 205.0658; found: 205.0662 .

Anal. Calcd for $\mathrm{C}_{12} \mathrm{H}_{12} \mathrm{NCl}$ (205.68): C, 70.07; H, 5.88; N, 6.81. Found: C, 69.99; H, 5.81; N, 6.76 .

\section{(E)-6-(2-Aminophenyl)-4-hexenenitrile (12)}

By adopting the described procedure, ${ }^{14 \mathrm{~b}}$ to an oven-dried, $38-\mathrm{mL}$ pressure tube equipped with a magnetic stir bar were added aryl chloride $11(737 \mathrm{mg}, 3.6 \mathrm{mmol})$ and $\left(\mathrm{NH}_{4}\right)_{2} \mathrm{SO}_{4}$ (710 mg, $5.4 \mathrm{mmol}, 1.5$ equiv), and then the tube was introduced into a glovebox. In the glovebox, in a separate oven-dried, 20-mL vial with a magnetic stir bar were added $\mathrm{Pd}[\mathrm{P}(2$ $\left.\mathrm{Tol}_{3}\right]^{23} 2$ (12.8 mg, $0.018 \mathrm{mmol}, 0.005$ equiv), Josiphos (CAS no. 158923-11-6; $9.9 \mathrm{mg}$, $0.018 \mathrm{mmol}, 0.005$ equiv), and dioxane $(1 \mathrm{~mL})$, and the mixture was stirred for $5 \mathrm{~min}$. To the pressure tube were added $\mathrm{NaO} t-\mathrm{Bu}(1.55 \mathrm{~g}, 16.1 \mathrm{mmol}, 4.5$ equiv), dioxane $(18 \mathrm{~mL}, 0.2 \mathrm{M})$, and the $\mathrm{Pd} / \mathrm{Josiphos}$ solution $(1 \mathrm{~mL})$. The pressure tube was tightly sealed with the screw cap and removed from the glovebox. The reaction mixture was heated to $100{ }^{\circ} \mathrm{C}$ and stirred for $12 \mathrm{~h}$. Then, the mixture was cooled to r.t. and diluted with EtOAc $(10 \mathrm{~mL})$. The resulting dark mixture was filtered through a pad of Celite and the filtrate was concentrated under reduced pressure $\left(30^{\circ} \mathrm{C}, 10 \mathrm{mmHg}\right)$ to yield a brown oil. Purification by silica gel flash chromatography (40 g, $25 \mathrm{~mm} \varnothing$; hexanes to hexanes/EtOAc, 9:1) afforded $274 \mathrm{mg}$ (41\%) of $\mathbf{1 2}$ as a pale yellow oil. An analytically pure sample was obtained by Kugelrohr distillation $\left(90{ }^{\circ} \mathrm{C} / 0.01 \mathrm{mmHg}\right.$ ), affording $236 \mathrm{mg}(35 \%)$ of $\mathbf{1 2}$ as a colorless oil; bp $90{ }^{\circ} \mathrm{C} /$ $0.01 \mathrm{mmHg} ; R_{f}=0.41$ (hexanes/EtOAc, 4:1) [UV].

${ }^{1} \mathrm{H}$ NMR (500 MHz, $\left.\mathrm{CDCl}_{3}\right): \delta=7.11-7.02(\mathrm{~m}, 2 \mathrm{H}, \mathrm{HC}(10,12)), 6.77(\mathrm{t}, J=7.5 \mathrm{~Hz}, 1 \mathrm{H}$, $\mathrm{HC}(11)), 6.69$ (d, $J=7.5 \mathrm{~Hz}, 1 \mathrm{H}, \mathrm{HC}(9)), 5.71$ (dtd, $J=15.0,6.5,1.5 \mathrm{~Hz}, 1 \mathrm{H}, \mathrm{HC}(5)), 5.53$ (ddd, $J=15.0,6.5,4.5 \mathrm{~Hz}, 1 \mathrm{H}, \mathrm{HC}(4)), 3.63$ (br s, $2 \mathrm{H}, \mathrm{HN}$ ), 3.21 (dd, $J=6.5,1.5 \mathrm{~Hz}, 2 \mathrm{H}$, $\mathrm{HC}(6)), 2.46-2.35$ (m, $4 \mathrm{H}, \mathrm{HC}(2,3))$.

${ }^{13} \mathrm{C}$ NMR (126 MHz, $\mathrm{CDCl}_{3}$ ): $\delta=139.5$ (C7), 136.7 (C8), 130.4 (C5), $129.3(\mathrm{C} 12), 128.1$ (C4), 126.4 (C10), 119.1 (C11), 119.1 (C1), 115.1 (C9), 33.6 (C6), 28.4 (C3), 17.5 (C2).

MS (ESI): $m / z(\%)=103(17), 187(100)[\mathrm{M}+\mathrm{H}]^{+}, 209(26)$. 
HRMS (ESI): $m / z[M+H]^{+}$calcd for $\mathrm{C}_{12} \mathrm{H}_{15} \mathrm{~N}_{2}$ : 187.1235; found: 187.1232 .

Anal. Calcd for $\mathrm{C}_{12} \mathrm{H}_{14} \mathrm{~N}_{2}$ (186.12): C, 77.38; H, 7.58; N, 15.04. Found: C, 77.24; H, 7.41; $\mathrm{N}, 14.92$.

\section{(E)-N-[2-(5-Cyano-2-pentenyl)phenyl]-4-methylbenzenesulfon-amide (1i) ${ }^{11}$}

To an oven-dried, $25-\mathrm{mL}$, round-bottomed flask equipped with a magnetic stir bar were added aniline 12 (236 mg, $1.27 \mathrm{mmol}), \mathrm{CH}_{2} \mathrm{Cl}_{2}(2.5 \mathrm{~mL}, 0.5 \mathrm{M})$, pyridine ( $133 \mu \mathrm{L}, 1.65$ mmol, 1.3 equiv), and $\mathrm{TsCl}$ ( $266 \mathrm{mg}, 1.39 \mathrm{mmol}, 1.1$ equiv) in order at r.t. The reaction mixture was stirred for $24 \mathrm{~h}$, then washed with $1 \mathrm{M} \mathrm{HCl}$ solution $(3 \mathrm{~mL})$ and brine $(2 \times 3$ $\mathrm{mL}$ ). The resulting organic layer was dried over $\mathrm{Na}_{2} \mathrm{SO}_{4}$, filtered, and concentrated under reduced pressure $\left(30^{\circ} \mathrm{C}, 10 \mathrm{mmHg}\right)$ to yield a yellow oil. The crude product was purified by silica gel flash chromatography $(25 \mathrm{~g}, 30 \mathrm{~mm} \varnothing$; hexanes to hexanes/EtOAc, 4:1) to afford $372 \mathrm{mg}(86 \%)$ of $\mathbf{1 i}$ as a yellow solid. An analytically pure sample was obtained by recrystallization of the solid (boiling EtOAc/pentane, 1:10; $15 \mathrm{~mL}$ ), affording $341 \mathrm{mg}(79 \%)$ of $\mathbf{1} \mathbf{i}$ as pale yellow crystals; $\mathrm{mp} 91-92{ }^{\circ} \mathrm{C}$ (sealed tube); $R_{f}=0.35$ (hexanes/EtOAc, 4:1) [UV].

${ }^{1} \mathrm{H} \mathrm{NMR}\left(500 \mathrm{MHz}, \mathrm{CDCl}_{3}\right): \delta=7.65(\mathrm{~d}, J=8.0 \mathrm{~Hz}, 2 \mathrm{H}, \mathrm{HC}(8)), 7.39(\mathrm{~d}, J=7.5 \mathrm{~Hz}, 1 \mathrm{H}$, $\mathrm{HC}(3)$ ), 7.22 (d, J= 7.5 Hz, $2 \mathrm{H}, \mathrm{HC}(9)), 7.19-7.09$ (m, $3 \mathrm{H}, \mathrm{HC}(\operatorname{aryl}), 6.47$ (br s, $1 \mathrm{H}, \mathrm{HN}$ ), 5.75 (dt, $J=15.0,6.0 \mathrm{~Hz}, 1 \mathrm{H}, \mathrm{HC}(13)), 5.59$ (ddd, $J=15.0,6.0,4.5 \mathrm{~Hz}, 1 \mathrm{H}, \mathrm{HC}(14)$ ), 3.23 (dd, $J=6.5,1.5 \mathrm{~Hz}, 2 \mathrm{H}, \mathrm{HC}(12)), 2.42$ (s, $3 \mathrm{H}, \mathrm{HC}(11)), 2.50-2.35$ (m, $4 \mathrm{H}, \mathrm{HC}(15,16)$ ).

${ }^{13} \mathrm{C}$ NMR (126 MHz, $\left.\mathrm{CDCl}_{3}\right): \delta=143.6(\mathrm{C} 10), 136.5$ (C7), $135.9(\mathrm{C} 2), 134.1(\mathrm{C} 1), 131.6$ (C14), 130.5 (C13), 129.8 (C6), 129.6 (C9), 127.1 (C4), 127.1 (C3), 127.0 (C8), 126.9 (C5), 119.0 (C17), 35.9 (C12), 28.4 (C15), 21.5 (C11), 17.4 (C16).

MS (EI): $m / z(\%)=105$ (23), 118 (67), 130 (20), 184 (27), 185 (40), 186 (100), 272 (15), $340(52)[\mathrm{M}]^{+}, 357(10), 363(11)$.

HRMS (EI): $m / z$ [M] $]^{+}$calcd for $\mathrm{C}_{19} \mathrm{H}_{20} \mathrm{~N}_{2} \mathrm{O}_{2} \mathrm{~S}: 340.1245$; found: 340.1248 .

Anal. Calcd for $\mathrm{C}_{19} \mathrm{H}_{20} \mathrm{~N}_{2} \mathrm{O}_{2} \mathrm{~S}$ (340.44): C, 67.03; H, 5.92; N, 8.23. Found: C, 67.17; H, $6.10 ; \mathrm{N}, 8.31$.

\section{(E)-4-Methyl-N-\{2-[6-(4-methylphenylsulfonamido)-2-hexen-yl]phenyl\}benzenesulfonamide}

(1j)

An oven-dried, 100-mL Schlenk flask equipped with a magnetic stir bar was charged with $\mathrm{LiAlH}_{4}(223 \mathrm{mg}, 5.87 \mathrm{mmol}, 2.0$ equiv) and capped with a septum under argon. The flask was immersed in an ice bath and THF $(10 \mathrm{~mL})$ was added. To the resulting suspension was added a solution of nitrile $\mathbf{1 i}(1.0 \mathrm{~g}, 2.94 \mathrm{mmol}, 1$ equiv) in THF $(10 \mathrm{~mL})$. The suspension was stirred for $2 \mathrm{~h}$ at $0{ }^{\circ} \mathrm{C}$ and gradually warmed to r.t. over $1 \mathrm{~h}$. The reaction was cooled to $0{ }^{\circ} \mathrm{C}$ and slowly quenched with aqueous $1 \mathrm{M} \mathrm{NaOH}$ solution $(0.4 \mathrm{~mL})$ upon completion. The resulting emulsion was filtered through a short pad of Celite and rinsed with $\mathrm{Et}_{2} \mathrm{O}(3 \times 15$ $\mathrm{mL})$. Concentration of the filtrate under reduced pressure $\left(25^{\circ} \mathrm{C}, 10 \mathrm{mmHg}\right)$ gave $799 \mathrm{mg}$ (79\%) of amine. Then, an oven-dried, $25-\mathrm{mL}$ Schlenk flask equipped with a magnetic stir 
bar was charged with the crude amine (689 mg, approx. $2.0 \mathrm{mmol}, 1$ equiv), $\mathrm{CH}_{2} \mathrm{Cl}_{2}$ (4 mL), and pyridine ( $210 \mu \mathrm{L}, 2.6 \mathrm{mmol}, 1.3$ equiv). To the solution was added $\mathrm{TsCl}$ ( $419 \mathrm{mg}, 2.2$ mmol, 1.1 equiv) portionwise at $0{ }^{\circ} \mathrm{C}$ under a positive stream of argon. The solution was warmed to r.t. and stirred for $4 \mathrm{~h}$. The reaction mixture was washed with aqueous $1 \mathrm{M} \mathrm{HCl}$ $(4 \mathrm{~mL})$, then brine $(2 \times 4 \mathrm{~mL})$. The resulting organic layer was dried over $\mathrm{Na}_{2} \mathrm{SO}_{4}$ and concentrated under reduced pressure $\left(25^{\circ} \mathrm{C}, 10 \mathrm{mmHg}\right)$. Purification by silica gel flash chromatography ( $40 \mathrm{~g}, 25 \mathrm{~mm} \varnothing$; hexanes to hexanes/EtOAc, 4:1) afforded $858 \mathrm{mg}$ (86\%) of the homologated tosylamine $\mathbf{1} \mathbf{j}$ as a white solid. An analytically pure sample was obtained by recrystallization of the solid (boiling EtOAc/pentane, 1:10; $30 \mathrm{~mL}$ ), affording $756 \mathrm{mg}$ (76\%) of $\mathbf{1 j}$ as white crystals; $\mathrm{mp} 104-105{ }^{\circ} \mathrm{C}$ (sealed tube); $R_{f}=0.28$ (hexanes/EtOAc, $4: 1$ ) [UV].

${ }^{1} \mathrm{H}$ NMR (500 MHz, $\left.\mathrm{CDCl}_{3}\right): \delta=7.74(\mathrm{~d}, J=8.0 \mathrm{~Hz}, 2 \mathrm{H}, \mathrm{HC}(19)), 7.65(\mathrm{~d}, J=8.0 \mathrm{~Hz}, 2$ $\mathrm{H}, \mathrm{HC}(8)), 7.39$ (d, $J=7.5 \mathrm{~Hz}, 1 \mathrm{H}, \mathrm{HC}(3)), 7.33$ (d, $J=8.0 \mathrm{~Hz}, 2 \mathrm{H}, \mathrm{HC}(20)), 7.22$ (d, $J=$ $7.5 \mathrm{~Hz}, 2 \mathrm{H}, \mathrm{HC}(9)), 7.19-7.09$ (m, $3 \mathrm{H}, \mathrm{HC}(\operatorname{aryl})$ ), 6.47 (br s, $1 \mathrm{H}, \mathrm{HN}$ ), 5.69 (dt, $J=15.0$, $6.5 \mathrm{~Hz}, 1 \mathrm{H}, \mathrm{HC}(13)), 5.54$ (dd, $J=15.0,7.0 \mathrm{~Hz}, 1 \mathrm{H}, \mathrm{HC}(14)), 4.65$ (br s, $1 \mathrm{H}, \mathrm{HN}$ ), 3.24 (dd, $J=6.5,1.5 \mathrm{~Hz}, 2 \mathrm{H}, \mathrm{HC}(12)), 2.92$ (app q, $J=7.0 \mathrm{~Hz}, 2 \mathrm{H}, \mathrm{HC}(17)), 2.43$ (s, $3 \mathrm{H}$, $\mathrm{HC}(22)$ ), 2.41 (s, $3 \mathrm{H}, \mathrm{HC}(11)$ ), 1.98 (app q, $J=7.0 \mathrm{~Hz}, 2 \mathrm{H}, \mathrm{HC}(15)$ ), 1.52 (app p, $J=7.0$ $\mathrm{Hz}, 2 \mathrm{H}, \mathrm{HC}(16))$.

${ }^{13} \mathrm{C}$ NMR (126 MHz, $\mathrm{CDCl}_{3}$ ): $\delta=143.6$ (C10), 143.3 (C21), 137.0 (C18), 136.5 (C7), 135.9 (C2), 134.1 (C1), 130.7 (C13), 129.9 (C14), 129.8 (C6), 129.7 (C20), 129.6 (C9), 127.1 (C4), 127.1 (C3), 127.1 (C19), 127.0 (C8), 126.9 (C5), 42.6 (C17), 35.8 (C12), 29.3 (C15), 29.2 (C16), 21.5 (C11), 21.5 (C22).

MS (ESI): $m / z(\%)=118(21), 273(13), 344$ (36), $499(100)[\mathrm{M}+\mathrm{H}]^{+}, 521(53), 537(24)$.

HRMS (ESI): $m / z[M+H]^{+}$calcd for $\mathrm{C}_{26} \mathrm{H}_{31} \mathrm{~N}_{2} \mathrm{O}_{4} \mathrm{~S}_{2}$ : 499.1725; found: 499.1728 .

Anal. Calcd for $\mathrm{C}_{26} \mathrm{H}_{30} \mathrm{~N}_{2} \mathrm{O}_{4} \mathrm{~S}_{2}$ (498.66): C, 62.62; H, 6.06; N, 5.62. Found: $\mathrm{C}, 62.85 ; \mathrm{H}$, $6.23 ; \mathrm{N}, 5.69$.

\section{(E)-1-Bromo-2-(3-heptenyl)benzene (17) (Scheme 10)}

To an oven-dried, 250-mL, round-bottomed flask equipped with a magnetic stir bar were added bromo alcohol $\mathbf{1 6}^{24}(3.21 \mathrm{~g}, 11.9 \mathrm{mmol})$ and $\mathrm{CH}_{2} \mathrm{Cl}_{2}(120 \mathrm{~mL}, 0.1 \mathrm{M})$, and then the flask was connected to an argon inlet. The solution was cooled to $0{ }^{\circ} \mathrm{C}$ in an ice bath, and $\mathrm{Et}_{3} \mathrm{~N}$ (5.83 mL, $41.8 \mathrm{mmol}, 3.5$ equiv) and $\mathrm{MsCl}$ (1.38 mL, $17.9 \mathrm{mmol}, 1.5$ equiv) were added by syringe. The reaction mixture was stirred for $1 \mathrm{~h}$ at $0{ }^{\circ} \mathrm{C}$. The reaction was quenched by adding $\mathrm{H}_{2} \mathrm{O}(30 \mathrm{~mL})$ and the resulting biphasic solution was separated. The aqueous layer was further extracted with $\mathrm{CH}_{2} \mathrm{Cl}_{2}(2 \times 30 \mathrm{~mL})$. The combined organic layer was dried over $\mathrm{Na}_{2} \mathrm{SO}_{4}$ and filtered over glass wool. The filtrate was concentrated under reduced pressure $\left(30^{\circ} \mathrm{C}, 10 \mathrm{mmHg}\right)$ to afford a brown oil. Purification by silica gel plug filtration (10 g, $30 \mathrm{~mm} \varnothing$; hexanes/EtOAc, 7:3) afforded $3.52 \mathrm{~g} \mathrm{(85 \% )}$ of the mesylate as a pale yellow oil. (The mesylate intermediate slowly turned into a dark oil upon standing.) To an oven-dried, 200-mL, round-bottomed flask with a magnetic stir bar were added the mesylate $(2.5 \mathrm{~g}, 7.2 \mathrm{mmol})$ and THF $(80 \mathrm{~mL}, 0.09 \mathrm{M})$. The flask was connected to an argon 
inlet and capped with a septum. The solution was cooled to $0{ }^{\circ} \mathrm{C}$ in an ice bath and a suspension of $\mathrm{LiAlH}_{4}(273 \mathrm{mg}, 7.2 \mathrm{mmol}, 1$ equiv) in THF $(10 \mathrm{~mL})$ was added by cannula dropwise. The resulting reaction mixture was stirred for $4 \mathrm{~h}$ at $0{ }^{\circ} \mathrm{C}$. The Fieser \& Fieser workup method was used to quench the reaction, adding $\mathrm{H}_{2} \mathrm{O}(0.3 \mathrm{~mL}), 15 \% \mathrm{NaOH}(0.3$ $\mathrm{mL})$, and $\mathrm{H}_{2} \mathrm{O}(0.9 \mathrm{~mL})$ in order, dropwise by syringe at $0{ }^{\circ} \mathrm{C}$. The resulting white slurry was filtered through Celite and rinsed with $\mathrm{Et}_{2} \mathrm{O}(2 \times 20 \mathrm{~mL})$. The colorless filtrate was concentrated under reduced pressure $\left(30^{\circ} \mathrm{C}, 10 \mathrm{mmHg}\right)$ to afford a colorless oil. Purification by silica gel flash chromatography $(120 \mathrm{~g}, 35 \mathrm{~mm} \varnothing$; hexanes to hexanes/EtOAc, 19:1) afforded $1.68 \mathrm{~g}(92 \%)$ of $\mathbf{1 7}$ as a colorless oil. An analytically pure sample was obtained by Kugelrohr distillation $\left(80^{\circ} \mathrm{C} / 0.1 \mathrm{mmHg}\right.$ ), affording $1.62 \mathrm{~g}(89 \%)$ of $\mathbf{1 7}$ as a colorless oil; bp $80{ }^{\circ} \mathrm{C} / 0.1 \mathrm{mmHg} ; R_{f}=0.67$ (hexanes/EtOAc, $4: 1$ ) [UV].

${ }^{1} \mathrm{H}$ NMR (500 MHz, $\left.\mathrm{CDCl}_{3}\right): \delta=7.53(\mathrm{~d}, J=8.0 \mathrm{~Hz}, 1 \mathrm{H}, \mathrm{HC}(6)), 7.25-7.17(\mathrm{~m}, 2 \mathrm{H}$, $\mathrm{HC}(3,4)), 7.07-7.02$ (m, $1 \mathrm{H}, \mathrm{HC}(5)), 5.46$ (q, $J=5.0 \mathrm{~Hz}, 2 \mathrm{H}, \mathrm{HC}(9,10)), 2.83-2.76$ (m, 2 H, HC(7)), 2.35-2.27 (m, 2 H, HC(8)), 1.97 (dt, $J=7.0,6.5 \mathrm{~Hz}, 2 \mathrm{H}, \mathrm{HC}(11)$ ), 1.36 (app sext, $J=7.5 \mathrm{~Hz}, 2 \mathrm{H}, \mathrm{HC}(12)), 0.88$ (t, $J=7.5 \mathrm{~Hz}, 3 \mathrm{H}, \mathrm{HC}(13))$.

${ }^{13} \mathrm{C}$ NMR (126 MHz, $\mathrm{CDCl}_{3}$ ): $\delta$ = 141.3 (C1), 132.7 (C6), 131.2 (C10), 130.4 (C3), 129.0 (C9), 127.4 (C5), 127.2 (C4), 124.4 (C2), 36.4 (C7), 34.7 (C11), 32.8 (C8), 22.6 (C12), 12.7 (C13).

MS (EI): $m / z(\%)=55$ (92), 67 (15), 82 (48), 83 (70), 89 (20), 90 (33), 91 (17), 117 (34), 169 (100), 171 (98), 173 (43), 182 (18), 184 (17), 252 (20) [M] $]^{+}, 254$ (20).

HRMS (EI): $\mathrm{m} / z$ [M] ${ }^{+}$calcd for $\mathrm{C}_{13} \mathrm{H}_{17} \mathrm{Br}$ : 252.0514; found: 252.0507 .

Anal. Calcd for $\mathrm{C}_{13} \mathrm{H}_{17} \mathrm{Br}$ (253.18): C, 61.67; H, 6.77. Found: C, 62.01; H, 6.69.

\section{(E)-2-(3-Heptenyl)aniline (18)}

By adopting the described procedure, ${ }^{14 \mathrm{~b}}$ to an oven-dried, 200-mL pressure tube equipped with a magnetic stir bar were added aryl bromide $17(1.52 \mathrm{~g}, 6.0 \mathrm{mmol})$ and $\left(\mathrm{NH}_{4}\right)_{2} \mathrm{SO}_{4}$ $(1.19 \mathrm{~g}, 9.0 \mathrm{mmol}, 1.5$ equiv), and then the tube was introduced into a glovebox. In the glovebox, in a separate oven-dried, $20-\mathrm{mL}$ vial with a magnetic stir bar were added $\mathrm{Pd}[\mathrm{P}(2-$ $\left.\mathrm{Tol})_{3}\right]_{2}{ }^{23}$ (21.5 mg, $0.03 \mathrm{mmol}, 0.005$ equiv), Josiphos (CAS no. 158923-11-6; $16.6 \mathrm{mg}$, $0.03 \mathrm{mmol}, 0.005$ equiv), and dioxane $(1 \mathrm{~mL})$, and the mixture was stirred for $5 \mathrm{~min}$. To the pressure tube were added $\mathrm{NaO} t$ - $\mathrm{Bu}(2.60 \mathrm{~g}, 27.0 \mathrm{mmol}$, 4.5 equiv), dioxane $(60 \mathrm{~mL})$, and the $\mathrm{Pd} / \mathrm{Josiphos} \mathrm{solution}(1 \mathrm{~mL})$. The pressure tube was tightly sealed with the screw cap and removed from the glove-box. The reaction mixture was heated to $100{ }^{\circ} \mathrm{C}$ and stirred for 12 h. Then, the mixture was cooled to r.t. and diluted with EtOAc $(30 \mathrm{~mL})$. The resulting dark mixture was filtered through a pad of Celite and the filtrate was concentrated under reduced pressure $\left(30^{\circ} \mathrm{C}, 10 \mathrm{mmHg}\right)$ to yield a brown oil. Purification by silica gel flash chromatography (120 g, $35 \mathrm{~mm} \varnothing$; hexanes to hexanes/EtOAc, 9:1) afforded $738 \mathrm{mg}$ (65\%) of $\mathbf{1 8}$ as a pale yellow oil. An analytically pure sample was obtained by Kugelrohr distillation $\left(110{ }^{\circ} \mathrm{C} / 0.1 \mathrm{mmHg}\right)$, affording $701 \mathrm{mg}(62 \%)$ of $\mathbf{1 8}$ as a colorless oil; bp $110^{\circ} \mathrm{C} / 0.1 \mathrm{mmHg} ; R_{f}=0.43$ (hexanes/EtOAc, $4: 1$ ) [UV]. 
${ }^{1} \mathrm{H} \mathrm{NMR}\left(500 \mathrm{MHz}, \mathrm{CDCl}_{3}\right): \delta=7.08-7.01(\mathrm{~m}, 2 \mathrm{H}, \mathrm{HC}(3,5)), 6.74(\mathrm{t}, J=7.5 \mathrm{~Hz}, 1 \mathrm{H}$, $\mathrm{HC}(4)), 6.68$ (d, $J=8.0 \mathrm{~Hz}, 1 \mathrm{H}, \mathrm{HC}(6)), 5.50$ (m, $2 \mathrm{H}, \mathrm{HC}(9,10)$ ), 3.62 (br s, $2 \mathrm{H}, \mathrm{NH}_{2}$ ), 2.55 (dd, $J=9.0,6.5 \mathrm{~Hz}, 2 \mathrm{H}, \mathrm{HC}(7)), 2.32$ (m, $2 \mathrm{H}, \mathrm{HC}(8)), 1.98$ (m, $2 \mathrm{H}, \mathrm{HC}(11)), 1.38$ (tq, $J=7.5,7.5 \mathrm{~Hz}, 2 \mathrm{H}, \mathrm{HC}(12)), 0.89$ (t, $J=7.5 \mathrm{~Hz}, 3 \mathrm{H}, \mathrm{HC}(13))$.

${ }^{13} \mathrm{C}$ NMR (126 MHz, $\left.\mathrm{CDCl}_{3}\right)$ : $\delta=144.1(\mathrm{C} 1), 131.1(\mathrm{C} 10), 129.5$ (C9 or C3), 129.4 (C3 or C9), 126.9 (C5), 126.3 (C2), 118.7 (C4), 115.5 (C6), 34.7 (C11), 31.8 (C8), 31.5 (C7), 22.6 (C12), 13.7 (C13).

MS (ESI): $m / Z(\%)=106(13), 190(100)[\mathrm{M}+\mathrm{H}]^{+}, 191(32)$.

HRMS (ESI): $\mathrm{m} / z[\mathrm{M}+\mathrm{H}]^{+}$calcd for $\mathrm{C}_{13} \mathrm{H}_{20} \mathrm{~N}$ : 190.1596; found: 190.1595 .

Anal. Calcd for $\mathrm{C}_{13} \mathrm{H}_{19} \mathrm{~N}$ (189.30): C, 82.48; H, 10.12; N, 7.40. Found: C, 82.48; H, 9.95; N, 7.69 .

\section{(E)-N-[2-(3-Heptenyl)phenyl]-4-methylbenzenesulfonamide (1k) ${ }^{11}$}

To an oven-dried, $25-\mathrm{mL}$, round-bottomed flask equipped with a magnetic stir bar were added aniline 18 (568 mg, $3.0 \mathrm{mmol}), \mathrm{CH}_{2} \mathrm{Cl}_{2}(6 \mathrm{~mL}, 0.5 \mathrm{M})$, pyridine $(315 \mu \mathrm{L}, 3.9 \mathrm{mmol}$, 1.3 equiv), and $\mathrm{TsCl}$ ( $686 \mathrm{mg}, 3.6 \mathrm{mmol}, 1.2$ equiv) in order at r.t. The reaction mixture was stirred for $12 \mathrm{~h}$, then washed with $1 \mathrm{M} \mathrm{HCl}$ solution $(5 \mathrm{~mL})$ and brine $(2 \times 5 \mathrm{~mL})$. The resulting organic layer was dried over $\mathrm{Na}_{2} \mathrm{SO}_{4}$, filtered, and concentrated under reduced pressure $\left(30{ }^{\circ} \mathrm{C}, 10 \mathrm{mmHg}\right)$ to yield a yellow oil. The crude product was purified by silica gel flash chromatography ( $80 \mathrm{~g}, 30 \mathrm{~mm} \varnothing$; hexanes to hexanes/EtOAc, 5:1) to afford 896 $\mathrm{mg}(87 \%)$ of $\mathbf{1 k}$ as a yellow oil which crystallized upon standing. An analytically pure sample was obtained by recrystallization of the solid (boiling EtOAc/pentane, 1:10;20 mL), affording $841 \mathrm{mg}(82 \%)$ of $1 \mathbf{k}$ as pale yellow crystals; $\mathrm{mp} 58-59{ }^{\circ} \mathrm{C}$ (sealed tube); $R_{f}=0.35$ (hexanes/EtOAc, 4:1) [UV].

${ }^{1} \mathrm{H} \mathrm{NMR}\left(500 \mathrm{MHz}, \mathrm{CDCl}_{3}\right): \delta=7.64(\mathrm{~d}, J=8.5 \mathrm{~Hz}, 2 \mathrm{H}, \mathrm{HC}(8)), 7.38(\mathrm{~d}, J=7.5 \mathrm{~Hz}, 1 \mathrm{H}$, $\mathrm{HC}(3)), 7.25$ (d, $J=7.5 \mathrm{~Hz}, 2 \mathrm{H}, \mathrm{HC}(9)), 7.20-7.10$ (m, $3 \mathrm{H}, \mathrm{HC}(\operatorname{aryl}), 6.51$ (br s, $1 \mathrm{H}, \mathrm{HN}$ ), 5.41-5.29 (m, $2 \mathrm{H}, \mathrm{HC}(14,15)), 2.42$ (s, $3 \mathrm{H}, \mathrm{HC}(11)), 2.41-2.35$ (m, $2 \mathrm{H}, \mathrm{HC}(12)), 2.10$ (q, $J=7.0 \mathrm{~Hz}, 2 \mathrm{H}, \mathrm{HC}(13)), 1.97$ (q, $J=6.5 \mathrm{~Hz}, 2 \mathrm{H}, \mathrm{HC}(16)), 1.38$ (sept d, $J=7.5,1.0 \mathrm{~Hz}, 2$ $\mathrm{H}, \mathrm{HC}(17)), 0.90(\mathrm{td}, J=7.5,1.0 \mathrm{~Hz}, 3 \mathrm{H}, \mathrm{HC}(18))$.

${ }^{13} \mathrm{C}$ NMR (126 MHz, $\left.\mathrm{CDCl}_{3}\right): \delta$ = 143.7 (C10), 136.6 (C7), $135.1(\mathrm{C} 2), 134.0(\mathrm{C} 1), 132.1$ (C15), 129.9 (C6), 129.6 (C9), 128.5 (C14), 127.1 (C8), 126.9 (C5), 126.2 (C4), 124.5 (C3), 34.6 (C16), 32.8 (C13), 30.9 (C12), 22.5 (C17), 21.5 (C11), 13.7 (C18).

MS (ESI): $m / z(\%)=118(25), 130$ (19), 132 (76), 146 (17), 187 (29), 188 (37), 189 (100), 190 (13), 205 (13), 286 (13), 342 (13), 344 (38) [M+ H] $]^{+}, 360$ (12), 366 (12).

HRMS (ESI): $m / z$ [M + H] $]^{+}$calcd for $\mathrm{C}_{20} \mathrm{H}_{26} \mathrm{NO}_{2} \mathrm{~S}: 344.1684$; found: 344.1680 .

Anal. Calcd for $\mathrm{C}_{20} \mathrm{H}_{25} \mathrm{NO}_{2} \mathrm{~S}$ (343.48): C, 69.93; H, 7.34; N, 4.08. Found: C, 69.89; H, $7.56 ; \mathrm{N}, 4.05$. 


\section{2-(4-Pentenyl)aniline (21) (Scheme 11)}

By adopting the described procedure, ${ }^{14 \mathrm{~b}}$ to an oven-dried, $250-\mathrm{mL}$ pressure tube equipped with a magnetic stir bar were added aryl bromide $\mathbf{2 0}^{18}(2.45 \mathrm{~g}, 10.9 \mathrm{mmol})$ and $\left(\mathrm{NH}_{4}\right)_{2} \mathrm{SO}_{4}$ $(2.16 \mathrm{~g}, 16.3 \mathrm{mmol}, 1.5$ equiv), and then the tube was introduced into a glovebox. In the glovebox, in a separate oven-dried, $20-\mathrm{mL}$ vial with a magnetic stir bar were added $\mathrm{Pd}[\mathrm{P}(2-$ $\left.\mathrm{Tol})_{3}\right]^{23} 2$ (38.9 mg, 0.054 mmol, 0.005 equiv), Josiphos (CAS no. 158923-11-6; $30.2 \mathrm{mg}$, $0.054 \mathrm{mmol}, 0.005$ equiv), and dioxane $(1 \mathrm{~mL})$, and the mixture was stirred for $5 \mathrm{~min}$. To the pressure tube were added $\mathrm{NaO} t$ - $\mathrm{Bu}(4.71 \mathrm{~g}, 49.0 \mathrm{mmol}, 4.5$ equiv), dioxane $(110 \mathrm{~mL})$, and the $\mathrm{Pd} / \mathrm{Josiphos} \mathrm{solution}(1 \mathrm{~mL})$. The pressure tube was tightly sealed with the screw cap and removed from the glovebox. The reaction mixture was heated to $100{ }^{\circ} \mathrm{C}$ and stirred for $12 \mathrm{~h}$. Then, the mixture was cooled to r.t. and diluted with EtOAc $(50 \mathrm{~mL})$. The resulting dark mixture was filtered through a pad of Celite and the filtrate was concentrated under reduced pressure $\left(30^{\circ} \mathrm{C}, 10 \mathrm{mmHg}\right)$ to yield a brown oil. Purification by silica gel flash chromatography (120 g, $35 \mathrm{~mm} \varnothing$; hexanes to hexanes/EtOAc, 6:1) afforded $1.07 \mathrm{~g} \mathrm{(61 \% )}$ of $\mathbf{2 1}$ as a pale yellow oil. An analytically pure sample was obtained by Kugelrohr distillation $\left(70{ }^{\circ} \mathrm{C} / 0.1 \mathrm{mmHg}\right)$, affording $1.01 \mathrm{~g}(57 \%)$ of 21 as a colorless oil; bp $70{ }^{\circ} \mathrm{C} / 0.1$ $\mathrm{mmHg} ; R_{f}=0.34$ (hexanes/EtOAc, $4: 1$ ) [UV].

${ }^{1} \mathrm{H}$ NMR (500 MHz, $\mathrm{CDCl}_{3}$ ): $\delta=7.07$ (td, $\left.J=7.5,1.5 \mathrm{~Hz}, 2 \mathrm{H}, \mathrm{HC}(3,5)\right), 6.76$ (tt, $J=7.5$, $1.5 \mathrm{~Hz}, 1 \mathrm{H}, \mathrm{HC}(4)), 6.70$ (d, $J=8.0 \mathrm{~Hz}, 1 \mathrm{H}, \mathrm{HC}(6)), 5.89$ (ddtd, $J=17.0,10.0,6.5,1.5 \mathrm{~Hz}$, $1 \mathrm{H}, \mathrm{HC}(10)), 5.09$ (dp, $J=17.0,1.5 \mathrm{~Hz}, 1 \mathrm{H}, \mathrm{HC}(11)), 5.04$ (dt, $J=10.0,1.5 \mathrm{~Hz}, 1 \mathrm{H}$, $\mathrm{HC}(11)), 3.62$ (br s, $2 \mathrm{H}, \mathrm{H}_{2} \mathrm{~N}$ ), 2.53 (t, $\left.J=8.0 \mathrm{~Hz}, 2 \mathrm{H}, \mathrm{HC}(7)\right), 2.18$ (dtd, $J=6.5,6.5,1.5$ $\mathrm{Hz}, 2 \mathrm{H}, \mathrm{HC}(9)$ ), 1.76 (qdd, $J=8.5,7.0,1.5 \mathrm{~Hz}, 2 \mathrm{H}, \mathrm{HC}(8)$ ).

${ }^{13} \mathrm{C}$ NMR (126 MHz, $\left.\mathrm{CDCl}_{3}\right): \delta$ = 143.9 (C1), 138.4 (C10), 129.5 (C3), 126.9 (C5), 126.5 (C2), 118.8 (C4), 115.6 (C6), 114.9 (C11), 33.5 (C9), 30.5 (C7), 27.8 (C8).

MS (ESI): $m / z(\%)=106(10), 162(100)[\mathrm{M}+\mathrm{H}]^{+}, 163(15), 174(10), 216(25)$.

HRMS (ESI): $m / z$ [M $+\mathrm{H}]^{+}$calcd for $\mathrm{C}_{11} \mathrm{H}_{16} \mathrm{~N}$ : 162.1283 ; found: 162.1288 .

Anal. Calcd for $\mathrm{C}_{11} \mathrm{H}_{15} \mathrm{~N}$ (161.24): C, 81.94; H, 9.38; N, 8.69. Found: C, 81.97; H, 9.13; N, 8.63 .

\section{4-Methyl-N-[2-(4-pentenyl)phenyl]benzenesulfonamide (1I) ${ }^{\mathbf{1 1}}$}

To an oven-dried, $25-\mathrm{mL}$, round-bottomed flask equipped with a magnetic stir bar were added aniline 21 (806 mg, $5.0 \mathrm{mmol}), \mathrm{CH}_{2} \mathrm{Cl}_{2}(10 \mathrm{~mL}, 0.5 \mathrm{M})$, pyridine $(526 \mu \mathrm{L}, 6.5 \mathrm{mmol}$, 1.3 equiv), and $\mathrm{TsCl}(1.14 \mathrm{~g}, 6.0 \mathrm{mmol}, 1.2$ equiv) in order at r.t. A condenser was installed on top of the flask and the reaction mixture was refluxed for $12 \mathrm{~h}$, then cooled to r.t. and washed with $1 \mathrm{M} \mathrm{HCl}$ solution $(10 \mathrm{~mL})$ and brine $(3 \times 5 \mathrm{~mL})$. The resulting organic layer was dried over $\mathrm{Na}_{2} \mathrm{SO}_{4}$, filtered, and concentrated under reduced pressure $\left(30^{\circ} \mathrm{C}, 10\right.$ $\mathrm{mmHg}$ ) to yield a yellow oil. The crude product was purified by silica gel flash chromatography (120 g, $35 \mathrm{~mm} \varnothing$; hexanes to hexanes/EtOAc, 5:1) to afford $1.34 \mathrm{~g} \mathrm{(85 \% )}$ of $\mathbf{1 1}$ as a white crystalline solid. An analytically pure sample was obtained by recrystallization of the solid (boiling pentane, $50 \mathrm{~mL}$ ), affording $1.23 \mathrm{~g}(78 \%)$ of $\mathbf{1 l}$ as white crystals; mp $70-71{ }^{\circ} \mathrm{C}$ (pentane); $R_{f}=0.30$ (hexanes/EtOAc, 4:1) [UV]. 
IR (neat): $3280(\mathrm{~m}), 1490(\mathrm{~m}), 1392(\mathrm{~m}), 1335$ (s), 1305 (w), 1291 (w), $1274(\mathrm{w}), 1185(\mathrm{w})$, 1160 (s), 1120 (m), 1092 (s), 1019 (w), 993 (w), 951 (w), $910(\mathrm{~s}), 886$ (w), 875 (w), $832(\mathrm{w})$, $814(\mathrm{~m}), 762(\mathrm{~m}) \mathrm{cm}^{-1}$.

${ }^{1} \mathrm{H}$ NMR $\left(500 \mathrm{MHz}, \mathrm{CDCl}_{3}\right): \delta=7.64(\mathrm{~d}, J=8.0 \mathrm{~Hz}, 2 \mathrm{H}, \mathrm{HC}(8)), 7.40(\mathrm{~d}, J=8.0 \mathrm{~Hz}, 1 \mathrm{H}$, $\mathrm{HC}(6)), 7.25$ (d, $J=8.0 \mathrm{~Hz}, 2 \mathrm{H}, \mathrm{HC}(9)), 7.21-7.11$ (m, $3 \mathrm{H}, \mathrm{HC}(3,4,5)), 6.36$ (br s, $1 \mathrm{H}$, HN), 5.78 (ddt, $J=17.0,10.5,6.5 \mathrm{~Hz}, 1 \mathrm{H}, \mathrm{HC}(15)$ ), 5.09-5.01 (m, $2 \mathrm{H}, \mathrm{HC}(16)), 2.43$ (s, 3 H, HC(11)), 2.34 (app t, $J=7.5 \mathrm{~Hz}, 2 \mathrm{H}, \mathrm{HC}(12)$ ), 2.01 (app q, $J=7.0 \mathrm{~Hz}, 2 \mathrm{H}, \mathrm{HC}(14)$ ), 1.49 (app p, $J=7.5 \mathrm{~Hz}, 2 \mathrm{H}, \mathrm{HC}(13)$ ).

${ }^{13} \mathrm{C}$ NMR (126 MHz, $\left.\mathrm{CDCl}_{3}\right): \delta=143.8(\mathrm{C} 10), 138.0(\mathrm{C} 15), 136.6(\mathrm{C} 7), 135.2(\mathrm{C} 1), 134.0$ (C2), 129.7 (C3), 129.6 (C9), 127.2 (C8), 126.9 (C4), 126.2 (C5), 124.5 (C6), 115.4 (C16), 33.1 (C14), 29.8 (C12), 29.0 (C13), 21.5 (C11).

MS (ESI): $m / z(\%)=160(15), 161(93), 162(16), 316(100)[\mathrm{M}+\mathrm{H}]^{+}, 317(20), 333(34)$, 335 (38), 338 (95), 339 (22), 492 (72), 493 (47).

HRMS (ESI): $m / z$ [M + H] $]^{+}$calcd for $\mathrm{C}_{18} \mathrm{H}_{22} \mathrm{NO}_{2} \mathrm{~S}: 316.1371$; found: 316.1376 .

Anal. Calcd for $\mathrm{C}_{18} \mathrm{H}_{21} \mathrm{NO}_{2} \mathrm{~S}$ (315.43): C, 68.54; H, 6.71; N, 4.44. Found: C, 68.43; H, $6.92 ; \mathrm{N}, 4.31$.

\section{Supplementary Material}

Refer to Web version on PubMed Central for supplementary material.

\section{Acknowledgments}

Mr. Zain Yousaf and Mr. Guanqun Zhang are thanked for assisting with substrate synthesis. Dr. David J. P. Kornfilt and Dr. Scott Barraza are thanked for providing helpful preparative advice and technical assistance.

Funding Information

We are grateful for generous financial support from the National Institutes of Health (R01 GM08525).

\section{References}

1. Denmark SE, Chi HM. J Org Chem. 2017; 82:3286.

2. (a) Sridharan V, Suryavanshi PA, Menéndez JC. Chem Rev. 2011; 111:7157. [PubMed: 21830756] (b) Katritzky AR, Rachwal S, Rachwal B. Tetrahedron. 1996; 52:15031.

3. For general reviews, see:(a) Nubbemeyer, U. The Claisen Rearrangement. Hiersemann, M., Nubbemeyer, U., editors. Wiley-VCH; Weinheim: 2007. Chap. 10b Nubbemeyer, U. Natural Products Synthesis II. Mulzer, J., editor. Springer; Berlin, Heidelberg: 2005. p. 149-213.(c) Majumdar KC, Bhattacharyya T, Chattopadhyay B, Sinha B. Synthesis. 2009:2117.(d) Lutz RP. Chem Rev. 1984; 84:205.

4. (a) Cooper MA, Lucas MA, Taylor JM, Ward AD, Williamson NM. Synthesis. 2001:621.(b) Anderson WK, Lai G. Synthesis. 1995:1287.(c) Takamatsu N, Inoue S, Kishi Y. Tetrahedron Lett. 1971:4661.(d) Krowicki K, Paillous N, Riviere M, Lattes A. J Heterocycl Chem. 1976; 13:555.(e) Jolidon S, Hansen HJ. Helv Chim Acta. 1977; 60:978.

5. (a) Sadownik JW, Philp D. Angew Chem Int Ed. 2008; 47:9965.(b) Grote RE, Jarvo ER. Org Lett. 2009; 11:485. [PubMed: 19093851] (c) Kozlov NG, Basalaeva LI. Russ J Gen Chem. 2001; 71:250. (d) Narasaka K, Shibata T. Heterocycles. 1993; 35:1039. 
6. Hatano M, Suzuki S, Ishihara K. J Am Chem Soc. 2006; 128:9998. [PubMed: 16881613]

7. Yip KT, Yang M, Law KL, Zhu NY, Yang D. J Am Chem Soc. 2006; 128:3130. [PubMed: 16522078]

8. Katritzky AR, Hong Q, Yang Z. J Org Chem. 1994; 59:7947.

9. (a) Chatterjee AK, Choi TL, Sanders DP, Grubbs RH. J Am Chem Soc. 2003; 125:11360. [PubMed: 16220959] (b) Koh MJ, Nguyen TT, Zhang H, Schrock RR, Hoveyda AH. Nature. 2016; 531:459. [PubMed: 27008965]

10. (a) Nicolaou KC, Bulger PG, Sarlah D. Angew Chem Int Ed. 2005; 44:4490.(b) Meek SJ, O’Brien RV, Llaveria J, Schrock RR, Hoveyda AH. Nature. 2011; 471:461. [PubMed: 21430774]

11. Yamamoto H, Ho E, Namba K, Imagawa H, Nishizawa M. Chem Eur J. 2010; 16:11271. [PubMed: 20730843]

12. Jiang F, Wu Z, Zhang W. Tetrahedron. 2011; 67:1501.

13. Denmark SE, Kornfilt DJP. J Org Chem. 2017; 82:3192. [PubMed: 28257203]

14. (a) Shekhar S, Dunn TB, Kotecki BJ, Mantavon DK, Cullen SC. J Org Chem. 2011; 76:4552. [PubMed: 21510695] (b) Green RA, Hartwig JF. Org Lett. 2014; 16:4388. [PubMed: 25133675] (c) Alsabeh PG, Lundgren RJ, McDonald R, Johansson Seechurn, CCC, Colacot TJ, Stradiotto M. Chem Eur J. 2013; 19:2131. [PubMed: 23293095]

15. (a) Choi, Y-M. Int Patent WO 2015/088271. 2015. p. 155-156.(b) Wang J, Chen J, Kee CW, Tan CH. Angew Chem Int Ed. 2012; 51:2382.(c) Grünanger CU, Breit B. Angew Chem Int Ed. 2008; 47:7346.

16. Nakhla JS, Kampf JW, Wolfe JP. J Am Chem Soc. 2006; 128:2893. [PubMed: 16506768]

17. Racouchot S, Sylvestre I, Ollivier J, Kozyrkov YY, Pukin A, Kulinkovich OG, Salaün J. Eur J Org Chem. 2002:2160.

18. Watson IDG, Ritter S, Toste FD. J Am Chem Soc. 2009; 131:2056. [PubMed: 19161306]

19. Arnold JS, Stone RF, Nguyen HM. Org Lett. 2010; 12:4580. [PubMed: 20843019]

20. Shu C, Leither A, Hartwig JF. Angew Chem Int Ed. 2004; 43:4797.

21. Muñiz K, Lishchynskyi A, Streuff J, Nieger M, Escudero-Adán EC, Martínez Belmonte, M. Chem Commun. 2011; 47:4911.

22. Yin Y, Zhao G. J Fluorine Chem. 2007; 128:40.

23. Harding BA, Melvin PR, Dougherty W Jr, Kassel S, Goodson FE. Organometallics. 2013; 32:3570.

24. Bruyère D, Bouyssi D, Balme G Tetrahedron. 2004; 60:4007. 

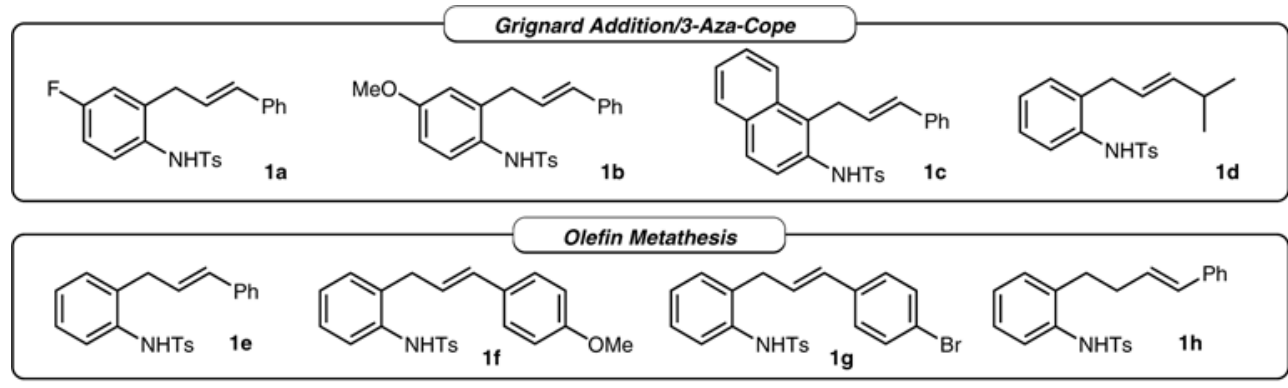

C-N Cross-Coupling

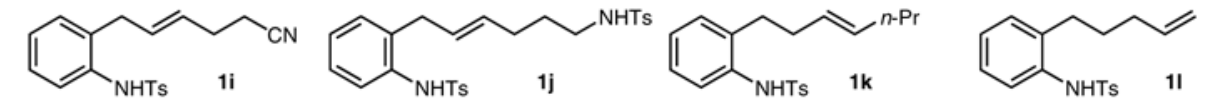

Figure 1.

Three approaches for the preparation of 2-alkenylanilines 

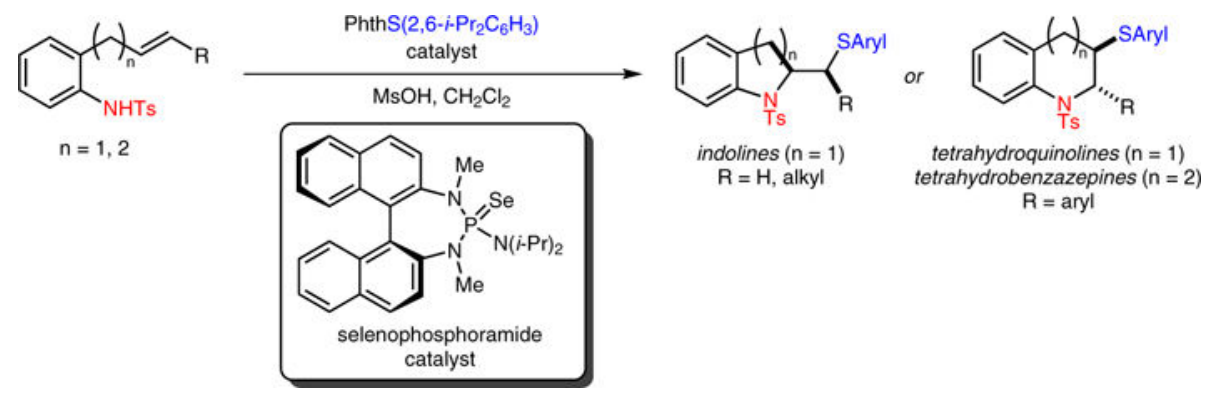

Scheme 1. 


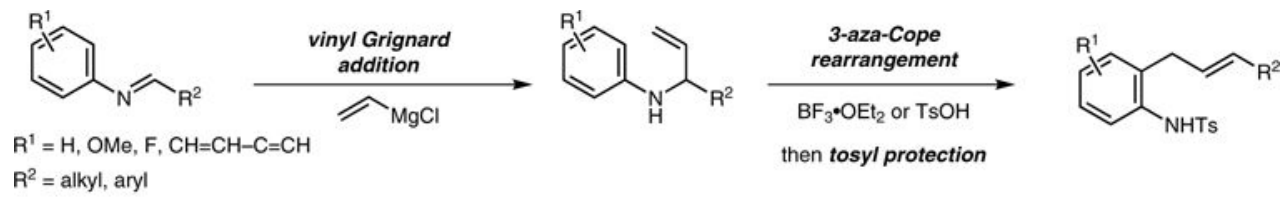
$\mathrm{R}^{2}=$ alkyl, aryl

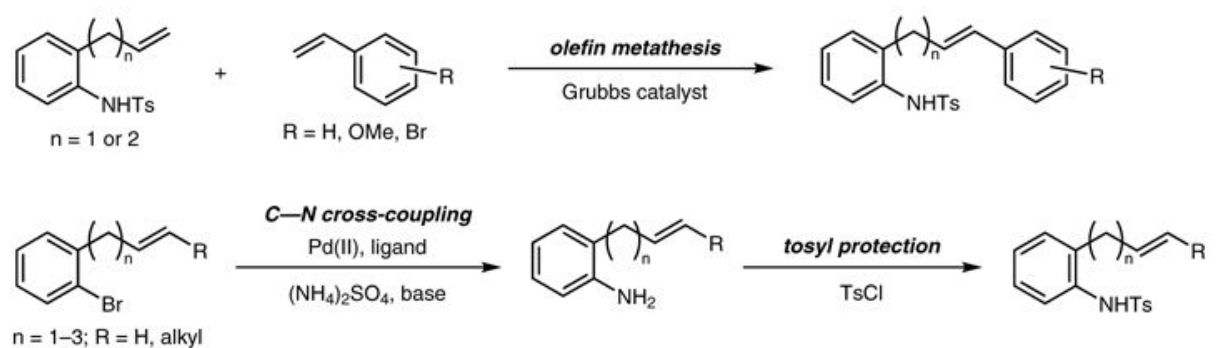

Scheme 2. 
<smiles>C1=CCCC=C1</smiles><smiles>C=C[C@H](O)OC(=O)O</smiles><smiles>C1=CCCC=C1</smiles><smiles>C=CCOC=C</smiles><smiles>C1CCCC1</smiles><smiles>C=CCCC=O</smiles><smiles>C=CCNC=C</smiles>

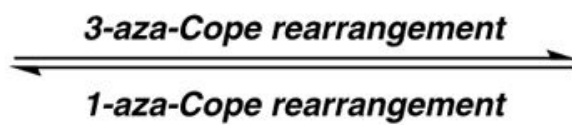<smiles>C=CCCC=N</smiles>

Scheme 3. 

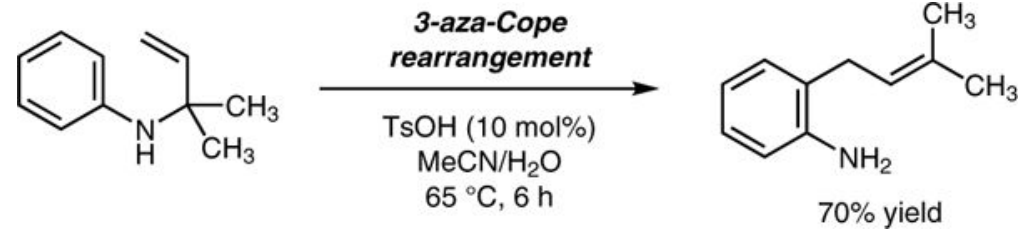

Scheme 4. 

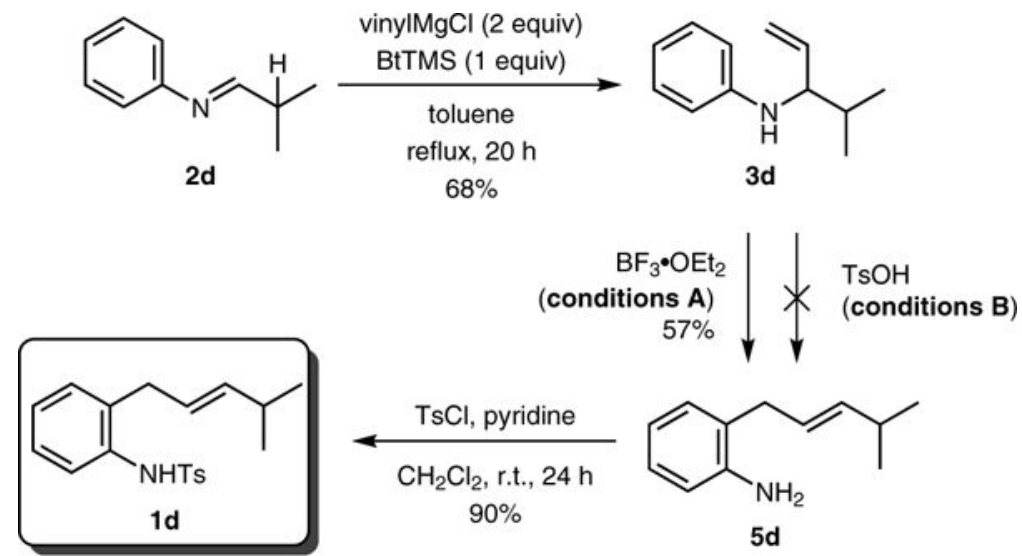

Scheme 5. 


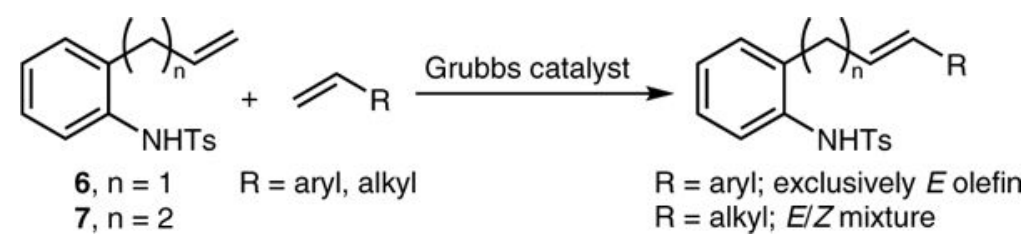

Scheme 6. 
a)

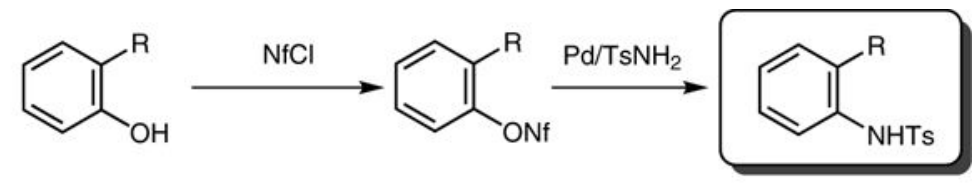

$\mathrm{R}=$ tethered olefin

b)

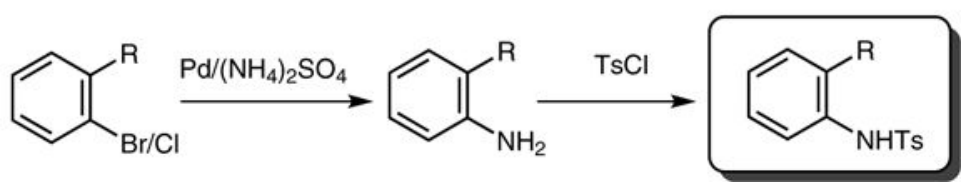

Scheme 7. 
<smiles>Cc1ccccc1ON</smiles>
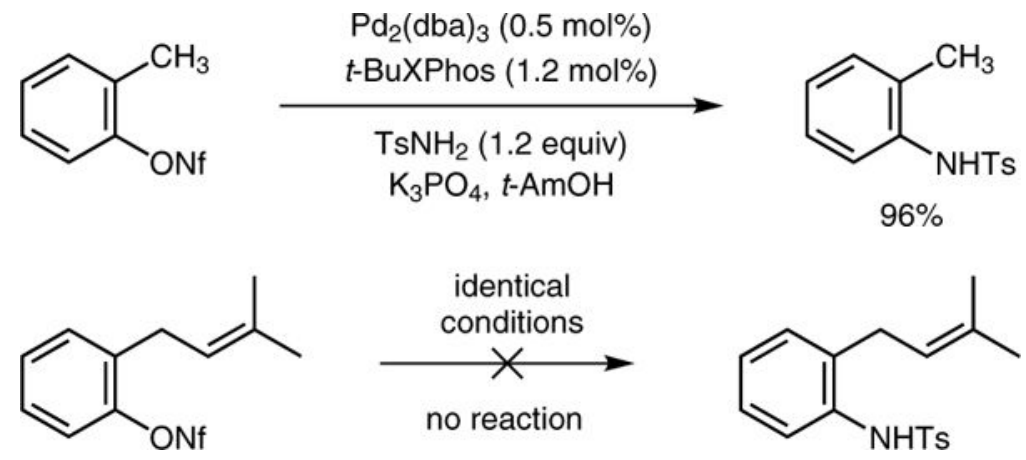

Scheme 8. 


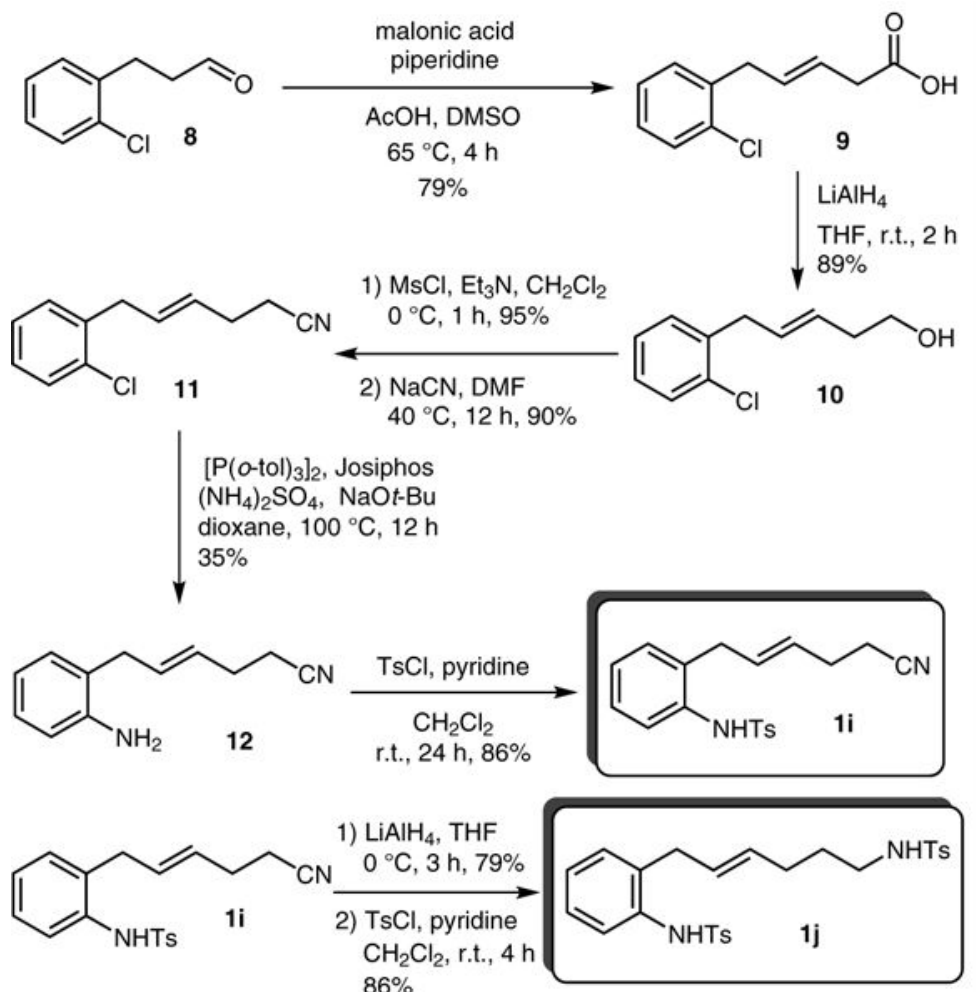

Scheme 9. 
<smiles>O=CCCc1ccccc1Br</smiles>

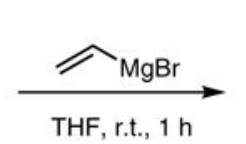<smiles>C=CC(O)CCc1ccccc1Br</smiles>

13

$(\mathrm{EtO})_{3} \mathrm{CMe}, \mathrm{EtCO}_{2} \mathrm{H}$ $140^{\circ} \mathrm{C}, 12 \mathrm{~h}$

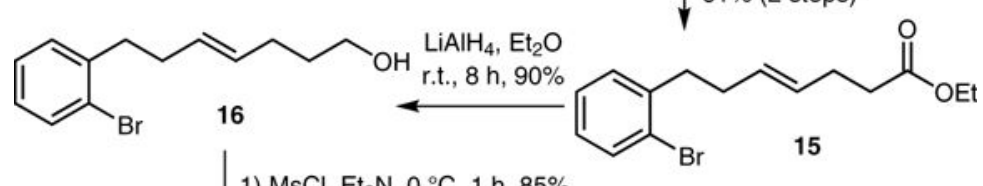

1) $\mathrm{MsCl}, \mathrm{Et}_{3} \mathrm{~N}, 0^{\circ} \mathrm{C}, 1 \mathrm{~h}, 85 \%$

2) $\mathrm{LiAlH}_{4}, \mathrm{THF}, 0^{\circ} \mathrm{C}, 4 \mathrm{~h}, 92 \%$
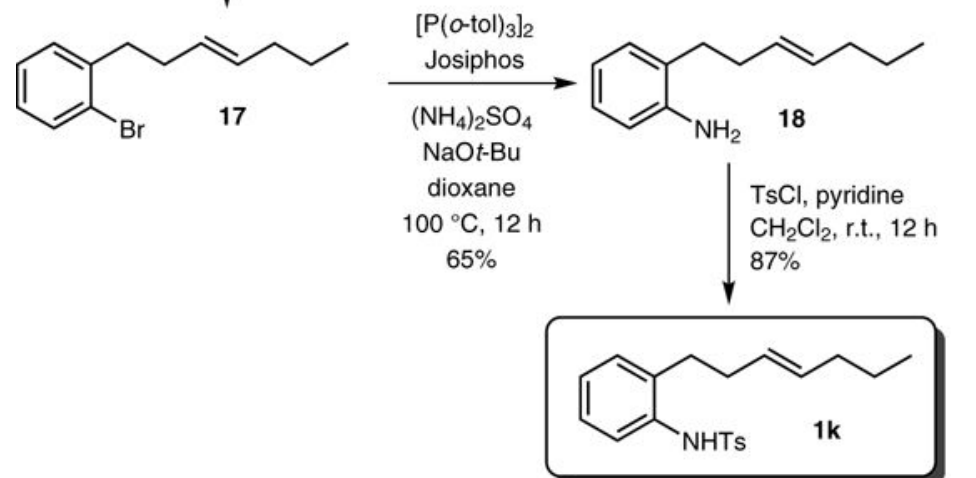

Scheme 10. 

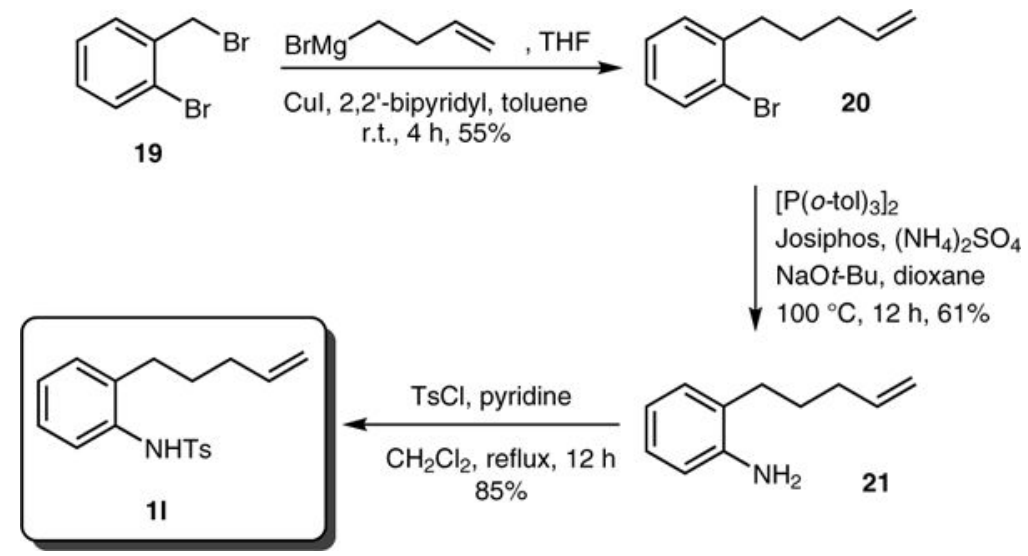

Scheme 11. 


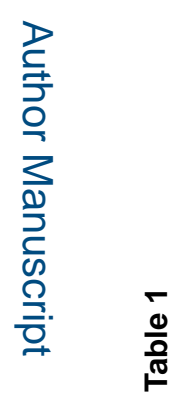

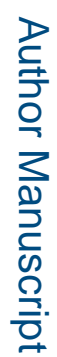

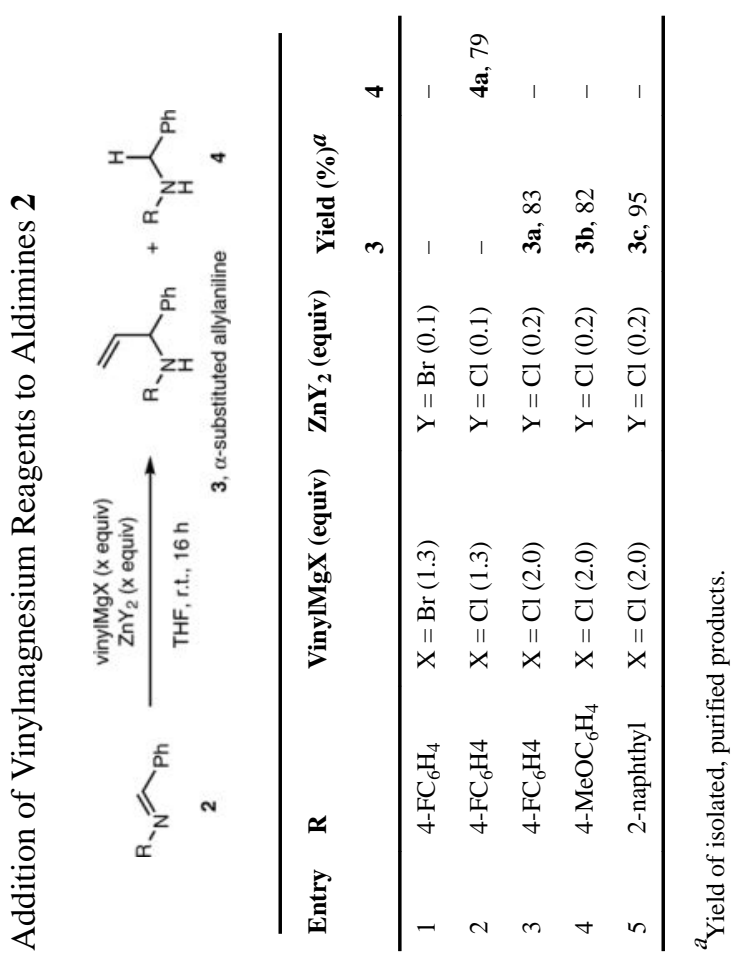

Synthesis (Stuttg). Author manuscript; available in PMC 2018 June 20. 


\section{를}

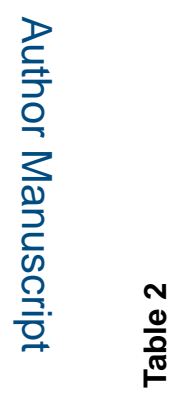

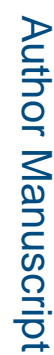

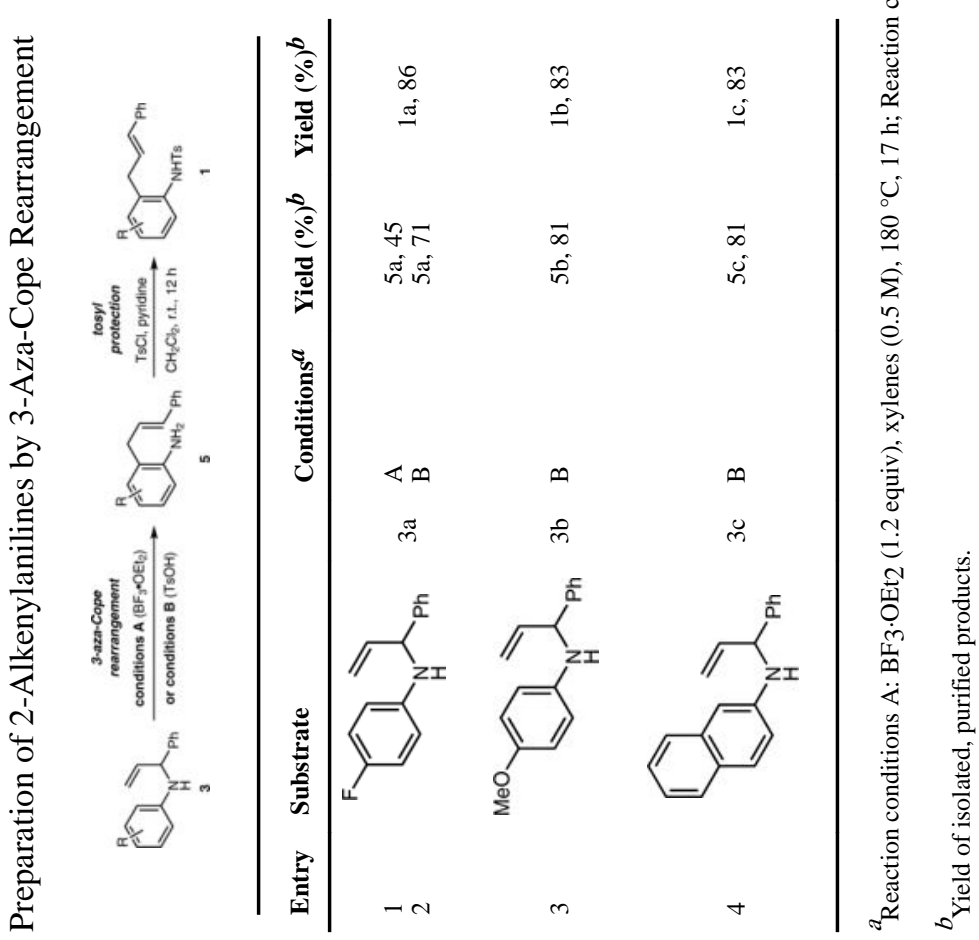

Synthesis (Stuttg). Author manuscript; available in PMC 2018 June 20. 


\section{로을}

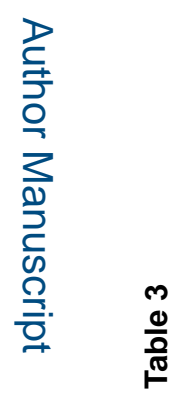

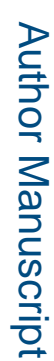

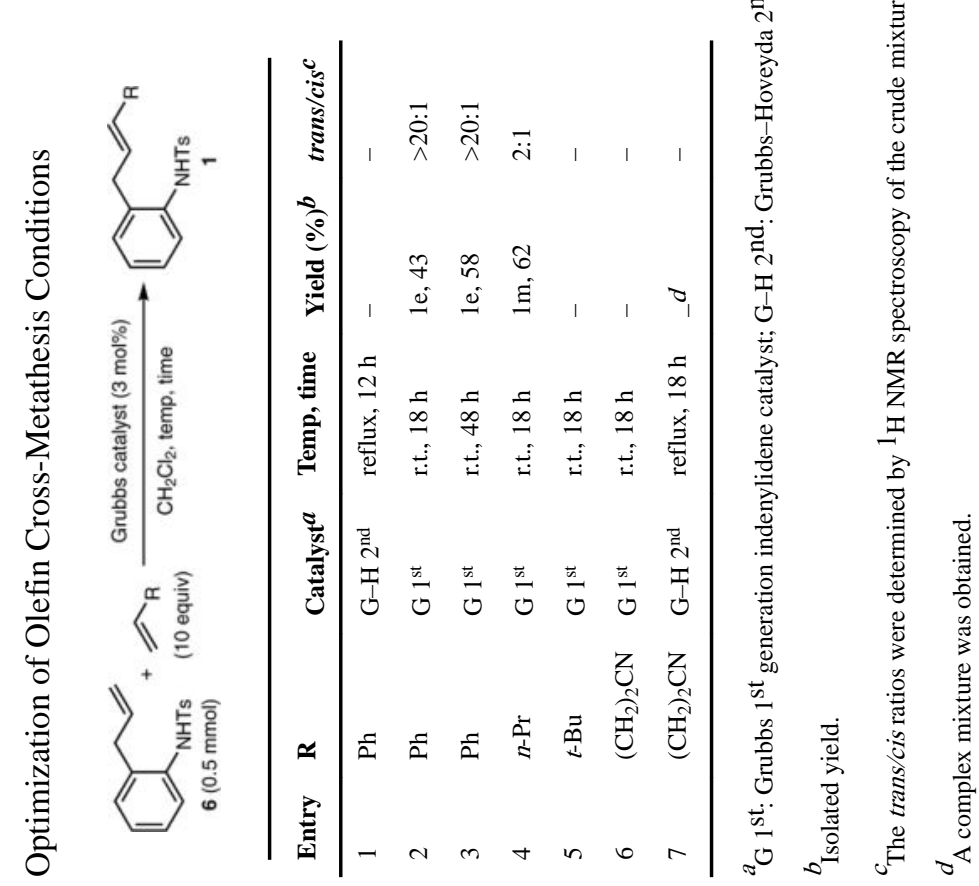

Synthesis (Stuttg). Author manuscript; available in PMC 2018 June 20. 


\section{룰}

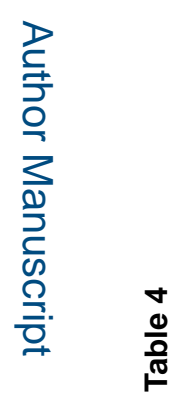

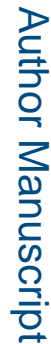

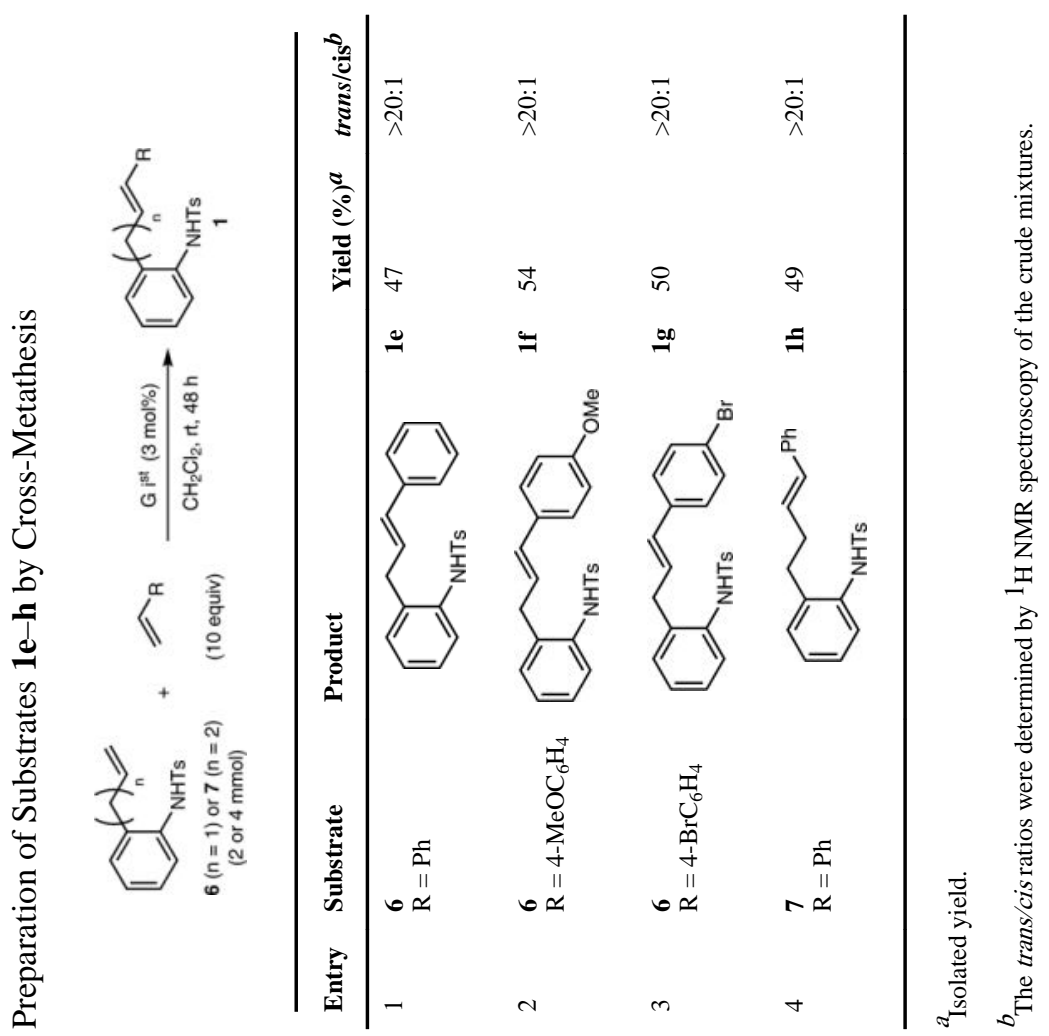

Synthesis (Stuttg). Author manuscript; available in PMC 2018 June 20. 